\title{
NUCLEATION OF BULK SUPERCONDUCTIVITY CLOSE TO CRITICAL MAGNETIC FIELD
}

\author{
SØREN FOURNAIS AND AYMAN KACHMAR
}

\begin{abstract}
We consider the two-dimensional Ginzburg-Landau functional with constant applied magnetic field. For applied magnetic fields close to the second critical field $H_{C_{2}}$ and large Ginzburg-Landau parameter, we provide leading order estimates on the energy of minimizing configurations. We obtain a fine threshold value of the applied magnetic field for which bulk superconductivity contributes to the leading order of the energy. Furthermore, the energy of the bulk is related to that of the Abrikosov problem in a periodic lattice. A key ingredient of the proof is a novel $L^{\infty}$-bound which is of independent interest.
\end{abstract}

\section{InTRODUCTION AND MAIN RESULTS}

Let us consider a two-dimensional, simply connected, open domain $\Omega \subset \mathbb{R}^{2}$ with smooth boundary. The energy of a cylindrical superconducting sample of cross section $\Omega$, placed in a constant applied magnetic field parallel to the cylinder axis, is given by the following GinzburgLandau functional:

$$
\begin{aligned}
\mathcal{E}(\psi, \mathbf{A} ; \Omega) & =\int_{\Omega} e_{\kappa, H}(\psi, \mathbf{A}) d x \\
& =\int_{\Omega}\left(|(\nabla-i \kappa H \mathbf{A}) \psi|^{2}-\kappa^{2}|\psi|^{2}+\frac{\kappa^{2}}{2}|\psi|^{4}+(\kappa H)^{2}|\operatorname{curl}(\mathbf{A}-\mathbf{F})|^{2}\right) \mathrm{d} x .
\end{aligned}
$$

Here $\psi$ is a complex valued wave function, $\mathbf{A}: \Omega \rightarrow \mathbb{R}^{2}$ a vector potential, $\kappa$ the Ginzburg-Landau parameter (a material parameter which is temperature independent), and $H$ is the strength of the applied magnetic field. The potential $\mathbf{F}: \Omega \rightarrow \mathbb{R}^{2}$ is the unique vector field satisfying,

$$
\operatorname{curl} \mathbf{F}=1, \quad \operatorname{div} \mathbf{F}=0 \quad \text { in } \Omega, \quad \nu \cdot \mathbf{F}=0 \quad \text { on } \partial \Omega,
$$

where $\nu$ is the unit inward normal vector of $\partial \Omega$.

In the last two decades, many authors have studied the minimization of the Ginzburg-Landau functional $\mathcal{E}$ in (1.1) over all admissible configurations $(\psi, \mathbf{A}) \in H^{1}(\Omega ; \mathbb{C}) \times H^{1}\left(\Omega ; \mathbb{R}^{2}\right)$. In the asymptotic limit $\kappa \rightarrow \infty$ (corresponding to type II superconductors), it is recognized that the behavior of the minimizers and their energy strongly depends on the applied magnetic field $H$. One distinguishes three different critical values $H_{C_{1}}, H_{C_{2}}$ and $H_{C_{3}}$ of the applied magnetic field that can be described roughly in the following way:

(1) If the applied magnetic field $H<H_{C_{1}}$, then $|\psi|$ does not vanish anywhere in $\Omega$, for any minimizer $(\psi, A)$ of the Ginzburg-Landau energy in (1.1).

(2) If $H_{C_{1}}<H<H_{C_{2}},|\psi|$ has isolated zeros in $\Omega$, called vortices.

(3) If $H_{C_{2}}<H<H_{C_{3}},|\psi|$ is small (in the bulk) except in a narrow region near the boundary of $\Omega$. This is the phenomenon called boundary superconductivity.

(4) If $H>H_{C_{3}},|\psi|$ vanishes everywhere in $\Omega$.

Precise mathematical definitions exist for the critical fields $H_{C_{1}}$ and $H_{C_{3}}$ which are precisely estimated in the limit $\kappa \rightarrow \infty$. We do not aim at giving an exhaustive list of references but we invite the reader to see the monographs [6, 13]. A mathematical definition of the critical field

Date: June 3, 2022. 
$H_{C_{2}}$ is still not available, but current mathematical results (c.f. [6, 12, 13, 14]) suggest that it behaves as follows in the large $\kappa$ regime,

$$
H_{C_{2}}=\kappa+o(\kappa) \quad \text { as } \kappa \rightarrow \infty .
$$

The present paper is devoted to a detailed analysis of the minimizers of the Ginzburg-Landau functional in the asymptotic regime $\kappa \rightarrow \infty$ and $H=\kappa+o(\kappa)$, which corresponds to type II superconductors subject to an applied magnetic field $H$ close to the critical field $H_{C_{2}}$. The obtained results are complementary to those in [4, 11, 12, 14].

1.1. Earlier results. The regime of applied magnetic fields close to the critical field $H_{C_{3}}$ is treated by Lu-Pan [10] (who, in particular, introduced a precise definition of this critical field), Helffer-Pan [9] and then by Fournais-Helffer [11]. This regime corresponds to applied magnetic fields $H=\frac{\kappa}{\Theta_{0}}+\rho(\kappa)$ where $\rho(\kappa)$ satisfies, $\lim _{\kappa \rightarrow \infty} \frac{\rho(\kappa)}{\kappa}=0$. The constant $\Theta_{0}$ appearing above is universal and satisfies $\Theta_{0} \in(0,1)$.

Among other things, the above mentioned papers give leading order estimates on the ground state energy,

$$
C_{0}(\kappa, H)=\inf _{(\psi, \mathbf{A}) \in H^{1}(\Omega ; \mathbb{C}) \times H^{1}\left(\Omega ; \mathbb{R}^{2}\right)} \mathcal{E}(\psi, \mathbf{A}) .
$$

Pan [12] and Almog-Helffer [4] give leading order estimates on the ground state energy, $C_{0}(\kappa, H)$ when $\kappa \rightarrow \infty$ and the applied magnetic field satisfies

$$
H=b \kappa+o(\kappa) \quad \text { as } \kappa \rightarrow \infty .
$$

The constant $b$ is assumed in the interval $\left[1, \Theta_{0}^{-1}\right.$ ) (with an extra condition when $b=1$, see Theorem 1.1 below). This regime corresponds to applied magnetic fields varying between the critical fields $H_{C_{2}}$ and $H_{C_{3}}$. Roughly speaking, the above mentioned papers show that the ground state energy satisfies,

$$
C_{0}(\kappa, H)=-C(b)|\partial \Omega| \kappa+o(\kappa) \quad \text { as } \kappa \rightarrow \infty,
$$

where $\left[1, \Theta_{0}^{-1}\right) \ni b \mapsto C(b) \in(0, \infty)$. The case $b=1$ corresponds to applied fields $H$ close to the critical field $H_{C_{2}}$. In strong connection with our results, we state the following theorem proved by Pan in [12], devoted to the case $b=1$. We use here the convention that a set $D \subset \Omega$ is smooth if there exists a smooth set $\widetilde{D} \subset \mathbb{R}^{2}$ such that $D=\widetilde{D} \cap \Omega$.

Theorem 1.1. There exists a positive universal constant $E_{1}$ such that, for any magnetic field $H=H(\kappa)$ satisfying,

$$
\frac{H}{\kappa} \rightarrow 1, \quad H-\kappa \rightarrow+\infty \quad \text { as } \kappa \rightarrow \infty,
$$

any minimizer $(\psi, \mathbf{A})$ of the energy $\mathcal{E}$ in (1.1) and any open, smooth domain $D \subset \Omega$, the following expansion holds

$$
\mathcal{E}(\psi, \mathbf{A} ; D)=-E_{1}|\bar{D} \cap \partial \Omega| \kappa+o(\kappa), \quad \text { as } \kappa \rightarrow \infty .
$$

Furthermore, Pan proves in [12] that $\psi$ decays away from the boundary $\partial \Omega$ in the $L^{2}$-sense (and this is actually one key ingredient to prove (1.5)) showing thus that the superconducting sample exhibits only surface superconductivity. A result by Almog [3] on the decay of $\psi$ permits one to extend the validity of Theorem 1.1 down to magnetic fields $H$ satisfying $H-\kappa \gg \frac{\ln \kappa}{\kappa}$ as $\kappa \rightarrow \infty$. Here we remind the reader that for two positive functions $a(\kappa)$ and $b(\kappa)$, the notation $a(\kappa) \ll b(\kappa)$ as $\kappa \rightarrow \infty$ means that $\lim _{\kappa \rightarrow \infty} \frac{a(\kappa)}{b(\kappa)}=0$.

The constant $E_{1}$ appearing in Theorem 1.1 is a universal constant defined via a reduced Ginzburg-Landau energy in a cylindrical domain. We will recall its definition in (2.10) below.

Complementary to the results of Pan [12], Sandier and Serfaty [14] consider the regime of magnetic fields

$$
H=b \kappa+o(\kappa) \quad \text { as } \kappa \rightarrow \infty,
$$


where the constant $b \in[0,1]$. Among other things, they prove that there exists a strictly increasing function $[0,1] \ni b \mapsto f(b) \in\left[-\frac{1}{2}, 0\right]$, with $f(1)=0$, such that the ground state energy satisfies

$$
C_{0}(\kappa, H)=f(b)|\Omega| \kappa^{2}+o\left(\kappa^{2}\right) \quad \text { as } \kappa \rightarrow \infty .
$$

More precisely, they prove a uniform energy density in $|\Omega|$ compatible with this global ground state energy. In the regime of interest to us, which corresponds to $b=1$, the ground state energy therefore satisfies,

$$
C_{0}(\kappa, H)=o\left(\kappa^{2}\right) \quad \text { as } \kappa \rightarrow \infty .
$$

We observe from the aforementioned results that a transition happens from bulk to boundary behavior when the applied field is close to $\kappa$, or in other words, when the applied field is close to the second critical field $H_{C_{2}}$. At the same time the order of magnitude of the energy changes here.

The results of the present paper (Theorem 1.2 below) determine the leading order term in the energy expansion (1.6), and indicates the optimal regime for the magnetic field $H$ such that Theorem 1.1 is valid. We obtain that the leading order behavior of the energy is determined according to variations of $H-\kappa$ on the critical scale $\sqrt{\kappa}$. Our results close the gap between the results of [12] and [14] and-taken together with the results of these papers-yield an overall understanding of the ground state energy of type II superconductors in strong magnetic fields.

1.2. Main results. In addition to the constant $E_{1}$ appearing in Theorem 1.1, the asymptotic behavior of the ground state energy $C_{0}(\kappa, H)$ involves another universal constant $E_{2}>0$. The definition of $E_{2}$ is related to the Abrikosov energy, see (2.12) and (2.24) below. We will use the function $\mathbb{R} \ni x \mapsto[x]_{+}:=\max (0, x)$.

Theorem 1.2. Let the positive constants $E_{1}$ and $E_{2}$ be defined by (2.10) and (2.12) respectively. Assume that the magnetic field satisfies,

$$
H=\kappa-\mu(\kappa) \sqrt{\kappa} \quad \text { such that } \quad \lim _{\kappa \rightarrow \infty} \frac{\mu(\kappa)}{\sqrt{\kappa}}=0 .
$$

Then, for any minimizer $(\psi, \mathbf{A})$ of the energy $\mathcal{E}$ in (1.1), and any open, smooth domain $D \subset \Omega$, the following asymptotic expansion holds,

$$
\mathcal{E}(\psi, \mathbf{A} ; D)=-E_{1}|\bar{D} \cap \partial \Omega| \kappa-E_{2}|D|[\mu(\kappa)]_{+}^{2} \kappa+o\left(\max \left(1,[\mu(\kappa)]_{+}^{2}\right) \kappa\right), \quad \text { as } \kappa \rightarrow \infty .
$$

Theorem 1.2 generalizes Theorem 1.1 and shows, in an energy sense, that the sample is in a surface superconducting state as long as the magnetic field satisfies $|H-\kappa| \ll \sqrt{\kappa}$ (see Corollary 1.3 for a qualitative statement on the behavior of order parameters $\psi$ ). In this specific regime, one difference between the proofs of Theorems 1.1 and 1.2 is that the order parameter $\psi$ is not expected to decay in the bulk. Hence we need a different method for controlling the energy contribution of the bulk, which we show to be negligible compared with that of the boundary.

However, as Theorem 1.2 shows, when the magnetic field strength $H$ becomes of the order $\kappa-\mu \sqrt{\kappa}$ with $\mu$ a positive constant, the energy contribution of the bulk can no more be neglected. Therefore, Theorem 1.1 gives a sharp description of how bulk superconductivity starts to appear, and thus establishes a fine characterization of the critical field $H_{C_{2}}$, which seems to be absent even in the Physics literature.

When the difference $\kappa-H$ becomes large compared with the critical scale $\sqrt{\kappa}$, Theorem 1.2 shows that the energy of the bulk becomes dominant to leading order.

As a corollary of Theorem 1.2, we get the following properties of the minimizing order parameter.

Corollary 1.3. Assume that the magnetic field satisfies,

$$
H=\kappa-\mu(\kappa) \sqrt{\kappa}, \quad \text { such that } \lim _{\kappa \rightarrow \infty} \frac{\mu(\kappa)}{\sqrt{\kappa}}=0 .
$$


Then for any minimizer $(\psi, \mathbf{A})$ of the energy $\mathcal{E}$ in (1.1), and any open, smooth domain $D \subset \Omega$, we have

$$
\kappa \int_{D}|\psi|^{4} d x=2\left(E_{1}|\bar{D} \cap \partial \Omega|+[\mu(\kappa)]_{+}^{2} E_{2}|D|\right)+o\left(\max \left(1,[\mu(\kappa)]_{+}^{2}\right)\right) .
$$

We conclude by stating a sharp $L^{\infty}$-bound in the following theorem. The motivation for this is twofold. Taken together with [5, Theorem 2.1], it is an affermative answer to a precise version of a conjecture by Sandier-Serfaty [14] and Aftalion-Serfaty [1]. It also plays a key-role in the proof of Theorem 1.2 announced above.

Theorem 1.4. Let $\delta \in(0,1)$ and $g: \mathbb{R}_{+} \rightarrow \mathbb{R}_{+}$be a function such that $g(\kappa) / \kappa \rightarrow 0$ as $\kappa \rightarrow \infty$. Then there exists a constant $C>0$ such that if $|H-\kappa| \leq g(\kappa)$, then

$$
\|\psi\|_{L^{\infty}\left(\omega_{\kappa}\right)} \leq C \lambda(\kappa)
$$

for all critical points $(\psi, \mathbf{A})$ of the energy in (1.1).

Here

$$
\omega_{\kappa}:=\left\{x \in \Omega \mid \operatorname{dist}(x, \partial \Omega) \geq \kappa^{-1+\delta}\right\}
$$

and

$$
\lambda(\kappa):=\max \left\{\left|\frac{\kappa}{H}-1\right|^{1 / 2}, \kappa^{-1+\delta}\right\} .
$$

In the regime of applied fields $H=\kappa-\mu(\kappa) \sqrt{\kappa}$ with $\lim _{\kappa \rightarrow \infty} \frac{\mu(\kappa)}{\sqrt{\kappa}}=0$ and $\lim _{\kappa \rightarrow \infty} \mu(\kappa)=\mu_{0} \in$ $(0,+\infty]$, the estimate of Theorem 1.4 is optimal. In this regime, the constant $\lambda(\kappa)$ above is equal to $\left|\frac{\kappa}{H}-1\right|^{1 / 2}$. It follows from Corollary 1.3 that there exists a constant $c>0$ such that, for any minimizer $(\psi, \mathbf{A})$, we have,

$$
c\left|\frac{\kappa}{H}-1\right|^{1 / 2} \leq\|\psi\|_{L^{\infty}\left(\omega_{\kappa}\right)} .
$$

The paper is organized as follows. Section 2 is devoted to some preliminaries, in particular, we recall some $a$ priori estimates together with the definitions of the universal constants $E_{1}$ and $E_{2}$. The proof of Theorem 1.4 is given in Section 3. In Sections 4 and 5, matching upper and lower bounds for the functional in (1.1) are obtained. Finally, Section 6 concludes with the proof of Theorem 1.2,

\section{Preliminaries}

2.1. A priori estimates. In this section, we collect some useful estimates for critical points of the Ginzburg-Landau functional $\mathcal{E}$ introduced in (1.1). The set of estimates in Lemma 2.2 appeared first in [10] (for a more particular regime) and were then proved for a wider regime in [6, 8]. The estimate of Lemma 2.3 was proved recently in [5].

Notice that a critical point $(\psi, \mathbf{A})$ of the functional $\mathcal{E}$ is a solution of the Ginzburg-Landau equations:

$$
\left\{\begin{array}{l}
-(\nabla-i \kappa H \mathbf{A})^{2} \psi=\kappa^{2}\left(1-|\psi|^{2}\right) \psi, \\
-\nabla^{\perp} \operatorname{curl} \mathbf{A}=(\kappa H)^{-1} \operatorname{Im}(\bar{\psi}(\nabla-i \kappa H \mathbf{A}) \psi), \quad \text { in } \Omega, \\
\nu \cdot(\nabla-i \kappa H \mathbf{A}) \psi=0, \quad \operatorname{curl} \mathbf{A}=1, \quad \text { on } \partial \Omega .
\end{array}\right.
$$

Here $\nu$ is the unit inward normal vector of $\partial \Omega$.

We start with the following direct consequence of the maximum principle.

Lemma 2.1. (13, Chapter 3]) Let $(\psi, \mathbf{A})$ be a solution of (2.1). Then $|\psi| \leq 1$ in $\bar{\Omega}$.

We also have elliptic estimates on the magnetic field and the energy density. 
Lemma 2.2. (Fournais-Helffer [8]) There exist positive constants $\kappa_{0}$ and $C$ such that, if the magnetic field satisfies $H \geq \frac{\kappa}{2}$ and if $(\psi, \mathbf{A})$ is a critical point of (1.1), then for all $\kappa \geq \kappa_{0}$, the following estimates hold,

$$
\begin{aligned}
& \|\operatorname{curl}(\mathbf{A}-\mathbf{F})\|_{C^{1}(\Omega)}+\kappa^{-1}\|\operatorname{curl}(\mathbf{A}-\mathbf{F})\|_{C^{2}(\Omega)} \leq C \kappa^{-1}, \\
& \|(\nabla-i \kappa H \mathbf{A}) \psi\|_{L^{\infty}(\Omega)} \leq C \kappa, \quad e_{\kappa, H}(\psi, \mathbf{A}) \leq C \kappa^{2} .
\end{aligned}
$$

Finally, close to $H_{C_{2}}$ the estimate of Lemma 2.1 can be improved.

Lemma 2.3. (Fournais-Helffer [5]) Assume that the magnetic field $H=H(\kappa)$ satisfies $\frac{H}{\kappa} \rightarrow 1$ as $\kappa \rightarrow \infty$. Then, given any function $g_{1}: \mathbb{R}_{+} \rightarrow(0,1]$ satisfying

$$
\lim _{\kappa \rightarrow \infty} g_{1}(\kappa)=0, \quad \lim _{\kappa \rightarrow \infty} \kappa g_{1}(\kappa)=\infty,
$$

there exists a function $g_{2}: \mathbb{R}_{+} \rightarrow(0,1]$ such that

$$
\lim _{\kappa \rightarrow \infty} g_{2}(\kappa)=0
$$

and

$$
\left.\left.\|\psi\|_{L^{\infty}(\{x \in \Omega}: \operatorname{dist}(x, \partial \Omega) \geq g_{1}(\kappa)\right\}\right) \leq g_{2}(\kappa) .
$$

2.2. The limiting boundary problem. We recall in this section the definition of the universal constant $E_{1}$ (appearing in Theorem 1.2 as given in [12]).

Let us consider the following magnetic potential (we keep the notation of [12]),

$$
\mathbf{E}(x)=\left(-x_{2}, 0\right), \quad \forall x=\left(x_{1}, x_{2}\right) \in \mathbb{R} \times \mathbb{R}_{+},
$$

together with the reduced Ginzburg-Landau energy,

$$
\mathcal{E}_{\ell}(\phi)=\int_{U_{\ell}}\left(|(\nabla-i \mathbf{E}) \phi|^{2}-|\phi|^{2}+\frac{1}{2}|\phi|^{4}\right) d x,
$$

where $U_{\ell}$ is the domain,

$$
U_{\ell}=(-\ell, \ell) \times(0, \infty), \quad \ell>0
$$

Let us introduce the space

$$
\mathcal{V}\left(U_{\ell}\right)=\left\{u \in L^{2}\left(U_{\ell}\right):(\nabla-i \mathbf{E}) u \in L^{2}\left(U_{\ell}\right), u( \pm \ell, \cdot)=0\right\} .
$$

We are interested in minimizing the energy (2.6) over the space $\mathcal{V}\left(U_{\ell}\right)$. So we introduce further,

$$
d(\ell)=\inf \left\{\mathcal{E}_{\ell}(\phi): \phi \in \mathcal{V}\left(U_{\ell}\right)\right\} .
$$

The following theorem is proved in [12, Theorems $4.4 \& 5.3]$.

Theorem 2.4. There exist positive constants $\ell_{0}, M$ and $E_{1}$ such that:

(1) For all $\ell \geq \ell_{0}$, (2.6) has a minimizer $\phi_{\ell}$ in $\mathcal{V}\left(U_{\ell}\right)$, and $\phi_{\ell} \not \equiv 0$.

(2) For all $\ell \geq \ell_{0}$, $\phi_{\ell}$ decays in the following way,

$$
\int_{U_{\ell} \cap\left\{x_{2} \geq 3\right\}} \frac{x_{2}^{2}}{\ln x_{2}}\left(\left|(\nabla-i \mathbf{E}) \phi_{\ell}\right|^{2}+\left|\phi_{\ell}\right|^{2}+x_{2}^{2}\left|\phi_{\ell}\right|^{4}\right) d x \leq M \ell .
$$

(3) For all $\ell \geq \ell_{0}$, the following estimate holds

$$
\left|\frac{d(\ell)}{2 \ell}+E_{1}\right| \leq \frac{M}{\ell}
$$

In light of Theorem 2.4, the universal constant $E_{1}>0$ is actually given as the limit,

$$
E_{1}=\lim _{\ell \rightarrow \infty}\left(-\frac{d(\ell)}{2 \ell}\right)
$$


2.3. The limiting bulk problem. We turn now to the limiting problem in the bulk, thereby defining the constant $E_{2}$ appearing in (1.7). Actually, $E_{2}$ can be defined in two different ways. The simpler definition is through a "thermodynamic limit" of the Ginzburg-Landau energy (see (2.12) ). A more complicated definition is via a limiting Abrikosov energy in a periodic lattice (see (2.24)). The latter approach in defining $E_{2}$ has more advantages, since on the one hand it shows rigorously how the Abrikosov energy links to the Ginzburg-Landau model, and on the other hand it provides an essential key for proving the main theorem of the present paper.

2.3.1. The universal constant $E_{2}$. Let us consider a constant $b \in(0,1)$. For any domain $\mathcal{D} \subset \mathbb{R}^{2}$, we define the following Ginzburg-Landau energy,

$$
G_{\mathcal{D}}(u)=\int_{\mathcal{D}} b\left|\left(\nabla-i \mathbf{A}_{0}\right) u\right|^{2}-|u|^{2}+\frac{1}{2}|u|^{4} d x .
$$

Here $\mathbf{A}_{0}$ is the canonical magnetic potential (we keep the notation from [1]),

$$
\mathbf{A}_{0}\left(x_{1}, x_{2}\right)=\frac{1}{2}\left(-x_{2}, x_{1}\right), \quad \forall x=\left(x_{1}, x_{2}\right) \in \mathbb{R}^{2} .
$$

It is proved by Sandier and Serfaty [14] (see also Aftalion-Serfaty [1, Lemma 2.4]) that there exists a continuous increasing function $g:(0,1] \rightarrow\left(-\frac{1}{2}, 0\right]$ such that the following identity holds,

$$
g(b)=\lim _{R \rightarrow \infty} \frac{\inf _{u \in H_{0}^{1}\left(K_{R} ; \mathbb{C}\right)} G_{K_{R}}(u, A)}{\left|K_{R}\right|},
$$

where $K_{R} \subset \mathbb{R}^{2}$ is a square of side-length equal to $R$. Furthermore, it is proved that there exists a constant $\alpha \in\left(0, \frac{1}{2}\right)$ such that

$$
\alpha(b-1)^{2} \leq|g(b)| \leq \frac{1}{2}(b-1)^{2}, \quad \forall b \in(0,1) .
$$

The universal constant $E_{2}$ is then defined by,

$$
E_{2}=\lim _{b \rightarrow 1_{-}} \frac{|g(b)|}{(b-1)^{2}} .
$$

The existence of the limit above is proved in [1, Theorem 2] and clearly satisfies

$$
0<\alpha \leq E_{2} \leq \frac{1}{2}
$$

Remark 2.5. For the sake of simplicity we considered only a square lattice above. This is because the lattice geometry is not important for the energy at this level. In [1], the results above are shown to be true for any parallelogram lattice and with the same constant $E_{2}$. This remark also applies to the remainder of the paper: We work with a square lattice as the basis for our constructions out of simplicity, and since this is known not to affect the energy to the precision considered.

Remark 2.6. Notice that the functional $G_{\mathcal{D}}$ can be rewritten, using the simple change of function $u=\sqrt{1-b} v$, as follows,

$$
G_{\mathcal{D}}(u)=(1-b)^{2}\left\{\frac{b}{1-b} \int_{\mathcal{D}}\left|\left(\nabla-i \mathbf{A}_{0}\right) v\right|^{2}-|v|^{2} d x+\int_{\mathcal{D}} \frac{1}{2}|v|^{4}-|v|^{2} d x\right\} .
$$

This simple manipulation provides a link between the Ginzburg-Landau energy $G_{\mathcal{D}}$ and the Abrikosov energy of Theorem 2.9 below. 
2.3.2. The periodic Schrödinger operator with constant magnetic field. Let $R>0$ and denote by $K_{R}$ the unit parallelogram of the lattice $\mathcal{L}_{R}=R(\mathbb{Z} \oplus i \mathbb{Z})$. We assume the quantization condition that $\left|K_{R}\right| /(2 \pi)$ is an integer, i.e. there exists $N \in \mathbb{N}$ such that,

$$
R^{2}=2 \pi N \text {. }
$$

Let us introduce the following space,

$$
\begin{aligned}
& E_{R}=\left\{u \in H^{1}\left(K_{R} ; \mathbb{C}\right):\right. u\left(z_{1}+R, z_{2}\right)=e^{i \frac{\pi N z_{2}}{R} u\left(z_{1}, z_{2}\right)} \\
&\left.u\left(z_{1}, z_{2}+R\right)=e^{-i \frac{\pi N z_{1}}{R}} u\left(z_{1}, z_{2}\right)\right\} .
\end{aligned}
$$

Recall the magnetic potential $\mathbf{A}_{0}$ introduced in (2.11) above. Notice that the periodicity conditions in (2.14) are constructed in such a manner that, for any function $u \in E_{R}$, the functions $|u|,\left|\nabla_{\mathbf{A}_{0}} u\right|$ and the vector field $\bar{u} \nabla_{\mathbf{A}_{0}} u$ are periodic with respect to the lattice $K_{R}$.

We denote by $P_{R}$ the operator,

$$
P_{R}=-\left(\nabla-i \mathbf{A}_{0}\right)^{2} \quad \text { in } L^{2}\left(K_{R}\right),
$$

with form domain the space $E_{R}$ introduced in (2.14). More precisely, $P_{R}$ is the self-adjoint realization associated with the closed quadratic form

$$
E_{R} \ni f \mapsto Q_{R}(f)=\left\|\left(\nabla-i \mathbf{A}_{0}\right) f\right\|_{L^{2}\left(K_{R}\right)}^{2} .
$$

The operator $P_{R}$ being with compact resolvent, let us denote by $\left\{\mu_{j}\left(P_{R}\right)\right\}_{j \geq 1}$ the increasing sequence of its distinct eigenvalues (i.e. without counting multiplicity).

The following proposition may be classical in the spectral theory of Schrödinger operators, but we refer to [1] or [2] for a simple proof.

Proposition 2.7. Assuming $R$ is such that $\left|K_{R}\right| \in 2 \pi \mathbb{N}$, then the operator $P_{R}$ enjoys the following spectral properties:

(1) $\mu_{1}\left(P_{R}\right)=1$ and $\mu_{2}\left(P_{R}\right) \geq 3$.

(2) The space $L_{R}=\operatorname{Ker}\left(P_{R}-1\right)$ is finite dimensional and $\operatorname{dim} L_{R}=\left|K_{R}\right| /(2 \pi)$.

Consequently, denoting by $\Pi_{1}$ the orthogonal projection on the space $L_{R}$ (in $L^{2}\left(K_{R}\right)$ ), and by $\Pi_{2}=\mathrm{Id}-\Pi_{1}$, then for all $f \in D\left(P_{R}\right)$, we have,

$$
\left\langle P_{R} \Pi_{2} f, \Pi_{2} f\right\rangle_{L^{2}\left(K_{R}\right)} \geq 3\|f\|_{L^{2}\left(K_{R}\right)}^{2} .
$$

The next lemma is a consequence of the existence of a spectral gap between the first two eigenvalues of $P_{R}$.

Lemma 2.8. Given $p \geq 2$, there exists a constant $C_{p}>0$ such that, for any $\gamma \in\left(0, \frac{1}{2}\right), R \geq 1$ with $\left|K_{R}\right| \in 2 \pi \mathbb{N}$, and $f \in D\left(P_{R}\right)$ satisfying

$$
Q_{R}(f)-(1+\gamma)\|f\|_{L^{2}\left(K_{R}\right)}^{2} \leq 0
$$

the following estimate holds,

$$
\left\|f-\Pi_{1} f\right\|_{L^{p}\left(K_{R}\right)} \leq C_{p} \sqrt{\gamma}\|f\|_{L^{2}\left(K_{R}\right)} .
$$

Here $\Pi_{1}$ is the projection on the space $L_{R}$.

Proof. Let us write $f_{1}=\Pi_{1} f$ and $f_{2}=f-\Pi_{1} f$, then since $f_{1}$ and $f_{2}$ are orthogonal we get $(\|\cdot\|$ denotes the $L^{2}$ norm unless otherwise stated),

$$
Q_{R}(f)=Q_{R}\left(f_{1}\right)+Q_{R}\left(f_{2}\right), \quad\|f\|^{2}=\left\|f_{1}\right\|^{2}+\left\|f_{2}\right\|^{2} .
$$

Furthermore, from (2.17) we deduce,

$$
\gamma\|f\|^{2} \geq Q_{R}\left(f_{1}\right)-\left\|f_{1}\right\|^{2}+Q_{R}\left(f_{2}\right)-\left\|f_{2}\right\|^{2}=Q_{R}\left(f_{2}\right)-\left\|f_{2}\right\|^{2} .
$$


Invoking Proposition 2.7 and the min-max variational principle, we infer the bound,

$$
\gamma\|f\|^{2} \geq \frac{1}{2} Q_{R}\left(f_{2}\right)+\frac{1}{2}\left\|f_{2}\right\|^{2} .
$$

Now, we claim that the following Sobolev inequality holds,

$$
\left\|f_{2}\right\|_{L^{p}\left(K_{R}\right)} \leq C_{p}\left(\left\|\nabla\left|f_{2}\right|\right\|_{L^{2}\left(K_{R}\right)}+\left\|f_{2}\right\|_{L^{2}\left(K_{R}\right)}\right),
$$

where $C_{p}>0$ is a constant independent of $R \in[1, \infty)$.

Using the diamagnetic inequality, we get further,

$$
\left\|f_{2}\right\|_{L^{p}\left(K_{R}\right)} \leq C_{p}\left(\sqrt{Q_{R}\left(f_{2}\right)}+\left\|f_{2}\right\|_{L^{2}\left(K_{R}\right)}\right) .
$$

By implementing (2.19) in the above estimate, we get the estimate announced in the lemma.

Thus, to finish the proof, we need only establish the estimate (2.20). Let $\chi$ be a cut-off function such that $0 \leq \chi \leq 1$ in $\mathbb{R}^{2}, \chi=1$ in $B(0,1)$ and $\operatorname{supp} \chi \subset B(0,2)$. Let further $C$ be a positive constant such that $B(0, C)$ contains $K_{1}$.

The function

$$
g(x)=\chi\left(\frac{x}{C R}\right)\left|f_{2}(x)\right|, \quad x \in \mathbb{R}^{2},
$$

belongs now to $H^{1}\left(\mathbb{R}^{2}\right)$. Using the Sobolev embedding of $H^{1}\left(\mathbb{R}^{2}\right)$ in $L^{p}\left(\mathbb{R}^{2}\right), p \geq 2$, we get a constant $c_{p}>0$ such that

$$
\|g\|_{L^{p}\left(\mathbb{R}^{2}\right)} \leq c_{p}\left(\|\nabla g\|_{L^{2}\left(\mathbb{R}^{2}\right)}+\|g\|_{L^{2}\left(\mathbb{R}^{2}\right)}\right) .
$$

Since the function $\left|f_{2}\right|$ is periodic with respect to the lattice $K_{R}$, and since

$$
\|\nabla g\|_{L^{2}\left(\mathbb{R}^{2}\right)}^{2} \leq 2\left\|\nabla\left|f_{2}\right|\right\|_{L^{2}(B(0, C R)}+\frac{2}{C^{2} R^{2}}\left\|f_{2}\right\|_{L^{2}(B(0, C R)}^{2},
$$

we get the estimate in (2.20).

2.3.3. The Abrikosov energy. Let us now introduce the following energy functional (the Abrikosov energy),

$$
F_{R}(v)=\frac{1}{\left|K_{R}\right|} \int_{K_{R}}\left(\frac{1}{2}|v|^{4}-|v|^{2}\right) d x .
$$

The energy $F_{R}$ will be minimized on the space $L_{R}$, the eigenspace of the first eigenvalue of the periodic operator $P_{R}$,

$$
\begin{aligned}
L_{R} & =\left\{u \in E_{R}: P_{R} u=u\right\} \\
& =\left\{u \in E_{R}:\left(\partial_{x_{1}}+i \partial_{x_{2}}+\frac{1}{2}\left(x_{1}+i x_{2}\right)\right) u=0\right\} .
\end{aligned}
$$

The following theorem is proved in [1, Theorems $1 \& 2]$.

\section{Theorem 2.9. Setting}

$$
c(R)=\min \left\{F_{R}(u): u \in L_{R}\right\}
$$

then the limit

$$
\lim _{\substack{R \rightarrow \infty \\\left|K_{R}\right| /(2 \pi) \in \mathbb{N}}} c(R)
$$

exists and is expressed by the universal constant $E_{2}$ introduced in (2.12) as follows,

$$
E_{2}=\lim _{\substack{R \rightarrow \infty \\\left|K_{R}\right| /(2 \pi) \in \mathbb{N}}}[-c(R)] .
$$

We conclude by showing that (2.21) admits minimizers in (2.22). 
Proposition 2.10. Let $F_{R}$ be the energy introduced in (2.21). The infimum of $F_{R}$ over the (eigen-) space $L_{R}$ is achieved by a function $f_{R} \in L_{R}$.

Furthermore, there exist positive constants $R_{0}$ and $C$ such that, for all $\tau \in \mathbb{C} \backslash \mathbb{R}$ and $R \geq R_{0}$, we have the estimate,

$$
\frac{1}{\left|K_{R}\right|} \int_{K_{R}}\left|f_{R}\right|^{2} d x+\frac{1}{\left|K_{R}\right|} \int_{K_{R}}\left|f_{R}\right|^{4} d x \leq C .
$$

Proof. The functional $F_{R}$ is clearly continuous on the finite dimensional space $L_{R}$. By applying the Cauchy-Schwarz inequality twice, we notice that,

$$
\begin{aligned}
F_{R}(v) & \geq \frac{1}{\left|K_{R}\right|}\left(\frac{1}{2} \int_{K_{R}}|v|^{4} d x-\sqrt{\left|K_{R}\right|}\left(\int_{K_{R}}|v|^{4} d x\right)^{1 / 2}\right) \\
& \geq \frac{1}{\left|K_{R}\right|}\left(\frac{1}{4} \int_{K_{R}}|v|^{4} d x-10\left|K_{R}\right|\right), \quad \forall v \in L_{R} .
\end{aligned}
$$

Hence, $F_{R}$ is positive outside a compact set and therefore the the (negative) minimum exists in the finite dimensional space $L_{R}$.

Noticing that $F_{R}\left(f_{R}\right) \leq 0$, we get the estimate (2.25) from the aforementioned Cauchy-Schwarz inequality.

\section{The improved $L^{\infty}$-BOUnd}

This section is devoted to the proof of Theorem 1.4. Before we give the proof, we state the following corollary to Theorem 1.4, which will be a key-ingredient in proving Theorem 1.2,

Corollary 3.1. Assume that the magnetic field satisfies $H=\kappa+\nu$ with $|\nu| \ll \kappa$ as $\kappa \rightarrow \infty$. Then there exist constants $C>0$ and $\kappa_{0}>0$ such that

$$
\|\psi\|_{L^{2}(\Omega)} \leq C \zeta(\kappa), \quad \forall \kappa \geq \kappa_{0},
$$

for all critical points $(\psi, \mathbf{A})$ of the energy $\mathcal{E}$ in (1.1). Here

$$
\zeta(\kappa)=\max \left\{\left|1-\frac{\kappa}{H}\right|^{1 / 2}, \kappa^{-1 / 4}\right\} .
$$

Proof. Let $\delta=\frac{1}{2}$. We write,

$$
\begin{aligned}
\int_{\Omega}|\psi|^{2} d x & =\int_{\left\{\operatorname{dist}(x, \partial \Omega) \leq \kappa^{-1+\delta}\right\}}|\psi|^{2} d x+\int_{\left\{\operatorname{dist}(x, \partial \Omega) \geq \kappa^{-1+\delta}\right\}}|\psi|^{2} d x \\
& \leq c \kappa^{-1+\delta}\|\psi\|_{L^{\infty}(\Omega)}+C\|\psi\|_{L^{\infty}\left(w_{\kappa}\right)} .
\end{aligned}
$$

Here $C$ is a positive constant and $w_{\kappa}=\left\{x \in \Omega\right.$ : $\left.\operatorname{dist}(x, \partial \Omega) \geq \kappa^{-1+\delta}\right\}$. Invoking the bound $|\psi| \leq 1$ together with the estimate in Theorem 1.4 and our choice of $\delta=\frac{1}{2}$, we get for some new constant $C$,

$$
\int_{\Omega}|\psi|^{2} d x \leq C\left(\kappa^{-1 / 2}+\max \left\{\left|1-\frac{\kappa}{H}\right|, \kappa^{-1}\right\}\right),
$$

which is the bound we wanted to prove.

Now we proceed in proving Theorem 1.4 .

Proposition 3.2. Let $\delta \in(0,1)$. There exist positive constants $\kappa_{0}$ and $C$ such that if $H \geq \kappa / 2$, $\kappa \geq \kappa_{0}$, then

$$
\|\operatorname{curl} \mathbf{A}-1\|_{L^{\infty}(\Omega)} \leq C H^{-1}\left(\kappa^{-1+\delta}+\|\psi\|_{L^{\infty}\left(\omega_{\kappa}\right)}\right) .
$$


Proof. Since curl $\mathbf{A}=1$ on $\partial \Omega$, we get by integrating from the boundary and using the second Ginzburg-Landau equation in (2.1),

$$
\begin{aligned}
|\operatorname{curl} \mathbf{A}(x)-1| & \leq(\kappa H)^{-1}\left\{\kappa^{-1+\delta}+\operatorname{dist}(x, \partial \Omega)\|\psi\|_{L^{\infty}\left(\omega_{\kappa}\right)}\right\}\|\nabla-i \kappa H \mathbf{A} \psi\|_{L^{\infty}(\Omega)} \\
& \leq \frac{C}{H}\left\{\kappa^{-1+\delta}+\|\psi\|_{L^{\infty}\left(\omega_{\kappa}\right)}\right\},
\end{aligned}
$$

where we used (2.3) to get the second inequality.

Proof of Theorem 1.4.

We argue by contradiction. Assume that there exist sequences $\left\{\kappa_{n}\right\},\left\{H_{n}\right\}$ and a sequence of critical points $\left\{\left(\psi_{n}, \mathbf{A}_{n}\right)\right\}$ such that,

$$
\kappa_{n} \rightarrow \infty, \quad H_{n}=\kappa_{n}+\nu_{n}, \quad \text { where } \quad\left|\nu_{n}\right| \leq g\left(\kappa_{n}\right),
$$

and

$$
\lambda_{n}^{-1}\left\|\psi_{n}\right\|_{L^{\infty}\left(\omega_{n}\right)} \rightarrow \infty \quad \text { as } n \rightarrow \infty .
$$

Here we have simplified notation by defining

$$
\omega_{n}:=\omega_{\kappa_{n}}, \quad \lambda_{n}:=\lambda\left(\kappa_{n}\right) .
$$

Since $\lambda_{n} \geq \kappa_{n}^{-1+\delta}$, we have

$$
0<\lambda_{n}^{-1} 2^{-\kappa_{n}^{\delta / 2}} \leq\left(\kappa_{n}^{\delta / 2}\right)^{2\left(\frac{1}{\delta}-1\right)} 2^{-\kappa_{n}^{\delta / 2}} \rightarrow 0 \quad \text { as } n \rightarrow \infty .
$$

Let us pick $N_{0} \in \mathbb{N}$ sufficiently large such that, for all $n \geq N_{0}$ we have,

$$
\lambda_{n}^{-1}\left\|\psi_{n}\right\|_{L^{\infty}\left(\omega_{n}\right)} \geq 2 \quad \text { and } \quad \lambda_{n}^{-1} 2^{-\kappa_{n}^{\delta / 2}} \leq 1 .
$$

For $M>0$ and $n \in \mathbb{N}$, we define

$$
\omega_{M, n}=\left\{x \in \Omega: \operatorname{dist}\left(x, \omega_{n}\right) \leq \frac{M \kappa_{n}^{\delta / 2}}{2 \kappa_{n}}\right\} .
$$

We claim that for each $n \geq N_{0}$, there exists $M_{n} \in\left[0, \kappa_{n}^{\delta / 2}\right] \cap \mathbb{N}$ such that,

$$
\left\|\psi_{n}\right\|_{L^{\infty}\left(\omega_{M_{n}, n}\right)} \geq \frac{1}{2}\left\|\psi_{n}\right\|_{L^{\infty}\left(\omega_{M_{n}+1, n}\right)} .
$$

Otherwise, there exists $n \geq N_{0}$ such that for all $M \in\left[0, \kappa_{n}^{\delta / 2}\right]$ we have,

$$
\left\|\psi_{n}\right\|_{L^{\infty}\left(\omega_{M, n}\right)}<\frac{1}{2}\left\|\psi_{n}\right\|_{L^{\infty}\left(\omega_{M+1, n}\right)}
$$

Then, using the a priori bound $\left\|\psi_{n}\right\|_{\infty} \leq 1$, we get

$$
\left\|\psi_{n}\right\|_{L^{\infty}\left(\omega_{n}\right)} \leq\left(\frac{1}{2}\right)^{\kappa_{n}^{\delta / 2}} .
$$

But, since $n \geq N_{0}$, the above bound is impossible in light of (3.6). Therefore, (3.7) holds for some $M_{n} \in\left[0, \kappa_{n}^{\delta / 2}\right] \cap \mathbb{N}$.

We choose now $P_{n} \in \omega_{M_{n}, n}$ such that

$$
\left|\psi_{n}\left(P_{n}\right)\right|=\left\|\psi_{n}\right\|_{L^{\infty}\left(\omega_{M_{n}, n}\right)},
$$

and we define

$$
\Lambda_{n}:=\left|\psi_{n}\left(P_{n}\right)\right| .
$$

Then, by assumption,

- $\lambda_{n}^{-1} \Lambda_{n} \rightarrow \infty$ as $n \rightarrow \infty$.

- $\operatorname{dist}\left(P_{n}, \partial \Omega\right) \geq \frac{1}{2} \kappa_{n}^{-1+\delta}$.

- $\Lambda_{n} \geq \frac{1}{2}\left\|\psi_{n}\right\|_{L^{\infty}\left(B\left(P_{n}, \frac{1}{2} \kappa_{n}^{-1+\delta / 2}\right)\right)}$. 
Moreover, from Lemma 2.3 we know that $\Lambda_{n} \rightarrow 0$ as $n \rightarrow \infty$.

Define now the following re-scaled functions, on $|x| \leq \frac{1}{4} \kappa_{n}^{\delta / 2}$ :

$$
\begin{aligned}
& \widetilde{\varphi}_{n}(x)=\Lambda_{n}^{-1} e^{-i \sqrt{\kappa_{n} H_{n}} \mathbf{A}_{n}\left(P_{n}\right)} \psi_{n}\left(P_{n}+\frac{x}{\sqrt{\kappa_{n} H_{n}}}\right), \\
& \widetilde{\mathbf{a}}_{n}(x)=\sqrt{\kappa_{n} H_{n}}\left(\mathbf{A}_{n}\left(P_{n}+\frac{x}{\sqrt{\kappa_{n} H_{n}}}\right)-\mathbf{A}_{n}\left(P_{n}\right)\right) .
\end{aligned}
$$

Using Proposition 3.2 and the assumption on $\Lambda_{n}$, we know that

$$
\left|\operatorname{curl} \mathbf{A}_{n}-1\right| \leq \frac{C}{\kappa_{n}}\left(\kappa_{n}^{-1+\delta}+\left\|\psi_{n}\right\|_{L^{\infty}\left(\omega_{n}\right)}\right) \leq \frac{C}{\kappa_{n}}\left(\kappa_{n}^{-1+\delta}+\Lambda_{n}\right) \leq \frac{2 C}{\kappa_{n}} \Lambda_{n} .
$$

Therefore, with a new constant $C$,

$$
\left|\operatorname{curl} \widetilde{\mathbf{a}}_{n}-1\right|=\left|\left(\operatorname{curl} \mathbf{A}_{\mathbf{n}}\right)\left(P_{n}+\frac{x}{\sqrt{\kappa_{n} H_{n}}}\right)-1\right| \leq \frac{C}{\kappa_{n}} \Lambda_{n} .
$$

So we can choose a gauge function $g_{n}$ such that $\mathbf{a}_{n}:=\widetilde{\mathbf{a}}_{n}-\nabla g_{n}$ satisfies

$$
\left|\mathbf{a}_{n}(x)-\mathbf{A}_{0}(x)\right| \leq \frac{C|x|}{\kappa_{n}} \Lambda_{n} .
$$

with $\mathbf{A}_{0}$ is the magnetic potential introduced in (2.11) corresponding for unit constant magnetic field.

We define

$$
\varphi_{n}:=e^{-i g_{n}} \widetilde{\varphi}_{n}
$$

Using (3.16) we get

$$
\left|\mathbf{a}_{n}(x)-\mathbf{A}_{0}(x)\right| \leq \frac{C|x|}{\kappa_{n}} \Lambda_{n}, \quad\left|\mathbf{a}_{n}(x)+\mathbf{A}_{0}(x)\right| \leq|x| .
$$

Furthermore, by (2.3), we get $\left|\left(\nabla-i \mathbf{a}_{n}\right) \varphi_{n}\right| \leq C$, so combined with (3.18) we get

$$
\begin{array}{r}
\left|\nabla \varphi_{n}(x)\right| \leq C(1+|x|), \\
\left|\varphi_{n}(0)\right|=1, \quad\left|\varphi_{n}(x)\right| \leq 2,
\end{array}
$$

for all $|x| \leq \frac{1}{4} \kappa_{n}^{\delta / 2}$.

The equation for $\varphi_{n}$ is

$$
-\Delta \varphi_{n}-2 i \mathbf{a}_{n} \cdot \nabla \varphi_{n}+\left|\mathbf{a}_{n}\right|^{2} \varphi_{n}=\frac{\kappa_{n}}{H_{n}}\left(1-\Lambda_{n}^{2}\left|\varphi_{n}\right|^{2}\right) \varphi_{n},
$$

on $|x| \leq \frac{1}{4} \kappa_{n}^{\delta / 2}$. We reformulate this as

$$
\begin{aligned}
{\left[\left(-i \nabla+\mathbf{A}_{0}\right)^{2}-1\right] \varphi_{n}=} & 2 i\left(\mathbf{a}_{n}-\mathbf{A}_{0}\right) \nabla \varphi_{n}-\left(\mathbf{a}_{n}-\mathbf{A}_{0}\right)\left(\mathbf{a}_{n}+\mathbf{A}_{0}\right) \varphi_{n} \\
& +\left(\frac{\kappa_{n}}{H_{n}}-1-\Lambda_{n}^{2}\left|\varphi_{n}\right|^{2}\right) \varphi_{n} .
\end{aligned}
$$

By elliptic estimates we get, exactly as in [5, (2.16)-(2.20)], that there exists a function $\varphi_{\infty} \in$ $L^{\infty}\left(\mathbb{R}^{2}\right)$ such that, up to the extraction of a subsequence,

$$
\varphi_{n} \stackrel{n \rightarrow \infty}{\longrightarrow} \varphi_{\infty}
$$

holds in $C^{1}(K)$ on any compact subset $K \subset \mathbb{R}^{2}$. Moreover, $\varphi_{\infty}$ satisfies $\left|\varphi_{\infty}(0)\right|=1$ and

$$
\left[\left(-i \nabla+\mathbf{A}_{0}\right)^{2}-1\right] \varphi_{\infty}=0 \text { in } \mathbb{R}^{2} .
$$


Consider now a localization function $\chi \in C^{\infty}(\mathbb{R})$ with $\chi(t)=1$ for $t \leq 1 / 2, \chi(t)=0$ for $t \geq 1$, and define $\chi_{n}(x)=\chi\left(\kappa_{n}^{-\eta}|x|\right)$ for some $\eta \in\left(0, \frac{\delta}{2}\right)$. We have the following equation for $\chi_{n} \varphi_{n}$ :

$$
\begin{aligned}
{\left[\left(-i \nabla+\mathbf{A}_{0}\right)^{2}-\right.} & 1]\left(\chi_{n} \varphi_{n}\right) \\
= & \chi_{n}\left[\left(-i \nabla+\mathbf{A}_{0}\right)^{2}-1\right] \varphi_{n}-2 i\left(\nabla \chi_{n}\right) \cdot\left(-i \nabla+\mathbf{A}_{0}\right) \varphi_{n}-\left(\Delta \chi_{n}\right) \varphi_{n} \\
= & 2 i\left(\mathbf{a}_{n}-\mathbf{A}_{0}\right) \chi_{n} \nabla \varphi_{n}-\left(\mathbf{a}_{n}-\mathbf{A}_{0}\right)\left(\mathbf{a}_{n}+\mathbf{A}_{0}\right) \chi_{n} \varphi_{n} \\
& +\left(\frac{\kappa_{n}}{H_{n}}-1-\Lambda_{n}^{2}\left|\varphi_{n}\right|^{2}\right) \chi_{n} \varphi_{n}-2 i\left(\nabla \chi_{n}\right) \cdot\left(-i \nabla+\mathbf{A}_{0}\right) \varphi_{n}-\left(\Delta \chi_{n}\right) \varphi_{n} .
\end{aligned}
$$

We introduce the projector $\Pi_{0}$ on the lowest Landau level. This projector is given explicitly by the integral kernel,

$$
\Pi_{0}(x, y)=\frac{1}{2 \pi} e^{\frac{i}{2}\left(x_{1} y_{2}-x_{2} y_{1}\right)} e^{-\frac{1}{2}(x-y)^{2}},
$$

and is continuous on $L^{p}\left(\mathbb{R}^{2}\right)$ for all $p \in[2, \infty]$.

Fix a function $f \in C_{0}^{\infty}\left(\mathbb{R}^{2}\right)$. We will prove that

$$
\int_{\mathbb{R}^{2}}\left(\Pi_{0} f(x)\right)\left|\varphi_{\infty}(x)\right|^{2} \varphi_{\infty}(x) d x=0 .
$$

Since $f$ is arbitrary, this implies that

$$
\Pi_{0}\left(\left|\varphi_{\infty}\right|^{2} \varphi_{\infty}\right)=0 \quad \text { in } \mathbb{R}^{2} .
$$

But a result from [5] says that (3.26) combined with (3.23) implies that $\varphi_{\infty} \equiv 0$. This is in contradiction to the fact that $\left|\varphi_{\infty}(0)\right|=1$. Therefore we have reached a contradiction.

Thus, it only remains to prove (3.25).

By definition of $\Pi_{0}$ we have

$$
\int_{\mathbb{R}^{2}}\left(\Pi_{0} f(x)\right)\left[\left(-i \nabla+\mathbf{A}_{0}\right)^{2}-1\right]\left(\chi_{n} \varphi_{n}\right) d x=0 .
$$

By consequence,

$$
\lim _{n \rightarrow \infty} \Lambda_{n}^{-2} \int_{\mathbb{R}^{2}}\left(\Pi_{0} f(x)\right)\left[\left(-i \nabla+\mathbf{A}_{0}\right)^{2}-1\right]\left(\chi_{n} \varphi_{n}\right) d x=0 .
$$

We insert (3.24) in (3.28). Consider first the term with $\left(\frac{\kappa_{n}}{H_{n}}-1-\Lambda_{n}^{2}\left|\varphi_{n}\right|^{2}\right) \chi_{n} \varphi_{n}$. By assumption

$$
\Lambda_{n}^{-2}\left|\frac{\kappa_{n}}{H_{n}}-1\right| \leq \Lambda_{n}^{-2} \lambda_{n}^{2} \rightarrow 0 \quad \text { as } n \rightarrow \infty .
$$

So one readily gets the convergence,

$$
\Lambda_{n}^{-2} \int_{\mathbb{R}^{2}}\left(\Pi_{0} f(x)\right)\left(\frac{\kappa_{n}}{H_{n}}-1-\Lambda_{n}^{2}\left|\varphi_{n}\right|^{2}\right) \chi_{n} \varphi_{n} d x \stackrel{n \rightarrow \infty}{\longrightarrow}-\int_{\mathbb{R}^{2}}\left(\Pi_{0} f(x)\right)\left|\varphi_{\infty}\right|^{2} \varphi_{\infty} d x .
$$

So in order to obtain (3.25) we only have to prove that the other terms from (3.24) vanish in the limit.

By (3.18) and (3.19)

$$
\begin{aligned}
\mid \Lambda_{n}^{-2} \int_{\mathbb{R}^{2}}\left(\Pi_{0} f(x)\right) 2 i\left(\mathbf{a}_{n}-\mathbf{A}_{0}\right) \chi_{n} \nabla \varphi_{n} & -\left(\left(\mathbf{a}_{n}-\mathbf{A}_{0}\right)\left(\mathbf{a}_{n}+\mathbf{A}_{0}\right) \chi_{n} \varphi_{n} d x \mid\right. \\
& \leq \Lambda_{n}^{-2} \int_{\mathbb{R}^{2}}\left|\Pi_{0} f(x)\right| C \frac{\left(1+|x|^{2}\right) \Lambda_{n}}{\kappa_{n}} d x \\
& \rightarrow 0 \text { as } n \rightarrow \infty
\end{aligned}
$$

where we used the assumption that $\Lambda_{n} \gg \kappa_{n}^{-1}$ as $n \rightarrow \infty$.

Notice, using the compact support of $f$ and the off-diagonal decay of $\Pi_{0}$, that $\Pi_{0} f(x)$ is exponentially small on $\left\{|x| \geq \kappa^{-\eta}\right\}$. Therefore, it is easy to see that also

$$
\Lambda_{n}^{-2} \int_{\mathbb{R}^{2}}\left(\Pi_{0} f(x)\right)\left\{2 i\left(\nabla \chi_{n}\right) \cdot\left(-i \nabla+\mathbf{A}_{0}\right) \varphi_{n}+\left(\Delta \chi_{n}\right) \varphi_{n}\right\} d x \rightarrow 0 \quad \text { as } n \rightarrow \infty .
$$


This finishes the proof of (3.25) and therefore of Theorem 1.4

\section{UPPER BOUND OF THE ENERGY}

In this section we construct test configurations and compute their energies, obtaining thus upper bounds for the functional $\mathcal{E}$ in (1.1). Recall the definition of the ground state energy $C_{0}(\kappa, H)$ in (1.3). We will prove the following theorem.

Theorem 4.1. Assume that the magnetic field satisfies

$$
H=\kappa-\mu(\kappa) \sqrt{\kappa},
$$

with $\mu: \mathbb{R}_{+} \mapsto \mathbb{R}$ a function such that

$$
\lim _{\kappa \rightarrow \infty} \frac{\mu(\kappa)}{\sqrt{\kappa}}=0 .
$$

Then, as $\kappa \rightarrow \infty$, the following upper bound holds for the ground state energy introduced in (1.3),

$$
C_{0}(\kappa, H) \leq-E_{1}|\partial \Omega| \kappa-E_{2}|\Omega|[\mu(\kappa)]_{+}^{2} \kappa+o\left(\max \left(1,[\mu(\kappa)]_{+}^{2}\right) \kappa\right) .
$$

Here $E_{1}>0$ and $E_{2}>0$ are the constants introduced in (2.10) and (2.24) respectively.

\subsection{Boundary configuration.}

4.1.1. Boundary coordinates. In order to treat the surface (boundary) energy contribution, we shall frequently pass to a coordinate system valid in a tubular neighborhood of $\partial \Omega$. For more details on these coordinates, see for instance [․, Appendix F].

For a sufficiently small $t_{0}>0$, we introduce the open set

$$
\Omega\left(t_{0}\right)=\left\{x \in \mathbb{R}^{2}: \operatorname{dist}(x, \partial \Omega)<t_{0}\right\} .
$$

Let $s \mapsto \gamma(s)$ be the parametrization of $\partial \Omega$ by arc-length and $\nu(s)$ the unit inward normal of $\partial \Omega$ at $\gamma(s)$.

Define the transformation

$$
\Phi:\left[-\frac{|\partial \Omega|}{2}, \frac{|\partial \Omega|}{2}[\times]-t_{0}, t_{0}\left[\ni(s, t) \mapsto \gamma(s)+t \nu(s) \in \Omega\left(t_{0}\right)\right.\right.
$$

and extend it to $\mathbb{R} \times$ ] $-t_{0}, t_{0}$ [ by periodicity with respect to $s$. The resulting transformation becomes a local diffeomorphism whose Jacobian is $|D \Phi|=1-t k(s)$, where $k$ denotes the curvature of $\partial \Omega$. For $x \in \Omega\left(t_{0}\right)$, we put

$$
\Phi^{-1}(x)=(s(x), t(x))
$$

and we get in particular that

$$
t(x)=\operatorname{dist}(x, \partial \Omega) .
$$

Using the coordinate transformation $\Phi$, we associate to any function $u \in L^{2}(\Omega)$, a function $\widetilde{u}$ defined in $\left[-\frac{|\partial \Omega|}{2}, \frac{|\partial \Omega|}{2}\left[\times\left[0, t_{0}\right]\right.\right.$ by,

$$
\widetilde{u}(s, t)=u\left(\Phi^{-1}(s, t)\right),
$$

and we will use the symbol $U_{\Phi}$ for the operator that maps $u$ to $\widetilde{u}$. Notice also that the function $\widetilde{u}$ extends naturally to a $|\partial \Omega|$-periodic function in $s \in \mathbb{R}$.

We get then the following change of variable formulae.

Proposition 4.2. Let $u \in H^{1}\left(\Omega\left(t_{0}\right)\right)$ and $\mathbf{A} \in H^{1}\left(\Omega ; \mathbb{R}^{2}\right)$. We write $\widetilde{u}(s, t)=u(\Phi(s, t))$,

$$
\widetilde{\mathbf{A}}_{1}=\mathbf{A}_{1} \circ \Phi, \quad \widetilde{\mathbf{A}}_{2}=\mathbf{A}_{2} \circ \Phi, \quad g(s, t)=1-t k(s) .
$$

Then we have :

$$
\int_{\Omega\left(t_{0}\right)}|(\nabla-i \mathbf{A}) u|^{2} d x=\int_{-\frac{|\partial \Omega|}{2}}^{\frac{|\partial \Omega|}{2}} \int_{0}^{t_{0}}\left[[g(s, t)]^{-2}\left|\left(\partial_{s}-i \widetilde{\mathbf{A}}_{1}\right) \widetilde{u}\right|^{2}+\left|\left(\partial_{t}-i \widetilde{\mathbf{A}}_{2}\right) \widetilde{u}\right|^{2}\right] g(s, t) d t d s,
$$


and

$$
\int_{\Omega\left(t_{0}\right)}|u(x)|^{2} d x=\int_{-\frac{|\partial \Omega|}{2}}^{\frac{|\partial \Omega|}{2}} \int_{0}^{t_{0}}|\widetilde{u}(s, t)|^{2} g(s, t) d t d s .
$$

Recall the vector field $\mathbf{F}$ introduced in (1.2). Another feature of the coordinate system $(s, t)$ is that it permits us to express $\mathbf{F}$ in a more explicit form (up to a gauge transformation).

Let us introduce the following two subsets of $\Omega\left(t_{0}\right)$,

$$
U_{1}=\Phi^{-1}\left(\left[-\frac{|\partial \Omega|}{2}, 0\right) \times\left[0, t_{0}\right)\right), \quad U_{2}=\Phi^{-1}\left(\left[0, \frac{|\partial \Omega|}{2}\right) \times\left[0, t_{0}\right)\right) .
$$

Lemma 4.3. There exist two functions $\chi_{1} \in C^{2}\left(U_{1} ; \mathbb{R}\right)$ and $\chi_{2} \in C^{2}\left(U_{2} ; \mathbb{R}\right)$ such that upon setting

then we have in the $(s, t)$ coordinates,

$$
\mathbf{F}^{1}=\mathbf{F}+\nabla \chi_{1} \quad \text { in } U_{1}, \quad \mathbf{F}^{2}=\mathbf{F}+\nabla \chi_{2} \quad \text { in } U_{2},
$$

$$
\left(U_{\Phi} \mathbf{F}^{j}\right)(s, t)=\left(-t+k(s) \frac{t^{2}}{2}, 0\right) \quad \text { in } \Phi^{-1}\left(U_{j}\right), \quad j=1,2 .
$$

The gauge transformation in Lemma 4.3 can not be applied globally in $\Omega\left(t_{0}\right)$, or otherwise one has to add a geometric constant $\gamma_{0}$ in the expression of the obtained field, see [6, Lemma F.1.1]. In order to avoid the presence of such a geometric constant, we partition the domain into two different subsets and work seperately in each of them.

4.1.2. The test configuration. Let us introduce, for reasons of convenience that will become clear, the following small parameter,

$$
\varepsilon=\frac{1}{\sqrt{\kappa H}}
$$

Let, for $\ell>0, \phi_{\ell}$ be a minimizer of the reduced Ginzburg-Landau energy $\mathcal{E}_{\ell}$ from (2.6), see Theorem 2.4. We make the following choice of $\ell$,

$$
\ell=\frac{|\partial \Omega|}{4 \varepsilon}
$$

Define, for $(s, t) \in\left[-\frac{|\partial \Omega|}{2}, \frac{|\partial \Omega|}{2}\right)$,

$$
\varphi_{\rho, \varepsilon}(s, t)=\left\{\begin{array}{ccc}
\chi\left(\frac{t}{\varepsilon^{\rho}}\right) \phi_{\ell}\left(\frac{s}{\varepsilon}+\ell, \frac{t}{\varepsilon}\right), & \text { if } & -\frac{|\partial \Omega|}{2} \leq s<0, \\
\chi\left(\frac{t}{\varepsilon^{\rho}}\right) \phi_{\ell}\left(\frac{s}{\varepsilon}-\ell, \frac{t}{\varepsilon}\right), & \text { if } & 0 \leq s<\frac{|\partial \Omega|}{2} .
\end{array}\right.
$$

The parameter $\rho \in(0,1)$ is to be chosen later, and $\chi \in C_{0}^{\infty}(\mathbb{R})$ is a standard cut-off function satisfying,

$$
0 \leq \chi \leq 1 \text { in } \mathbb{R}, \quad \chi=1 \text { in }\left(-\frac{1}{2}, \frac{1}{2}\right), \quad \text { and } \operatorname{supp} \chi \subset[-1,1] .
$$

The function $\varphi_{\rho, \varepsilon}$ is clearly in $H^{1}$, since $\phi_{\ell}$ vanishes for $s= \pm \ell$. Using the coordinate transformation (4.2), we get from $\varphi_{\rho, \varepsilon}$ a test function in $\Omega$,

$$
\psi_{\rho, \varepsilon}^{\text {bnd }}(x)=\left(\mathbf{1}_{U_{1}}(x) e^{-i \kappa H \chi_{1}(x)}+\mathbf{1}_{U_{2}}(x) e^{-i \kappa H \chi_{2}(x)}\right) \varphi_{\rho, \varepsilon}\left(\Phi^{-1}(x)\right), \quad \forall x \in \Omega\left(t_{0}\right),
$$

and extended by 0 on $\Omega \backslash \Omega\left(t_{0}\right)$. Here the gauges $\chi_{1}$ and $\chi_{2}$ are introduced in Lemma 4.3 above.

Lemma 4.4. Given two positive constants $m$ and $M$ with $m<M$, there exist positive constants $\varepsilon_{0}$ and $C$ such that if the magnetic field satisfies $m \kappa \leq H \leq M \kappa$, then for all $\varepsilon \in\left(0, \varepsilon_{0}\right], \rho \in(0,1)$ and $\delta \in(0,1)$, the following estimate holds,

$$
\mathcal{E}\left(\psi_{\rho, \varepsilon}^{\text {bnd }}, \mathbf{F} ; \Omega\right) \leq 2 d(\ell)+C\left(\varepsilon^{4-5 \rho}+\varepsilon^{\rho / 2}+\varepsilon^{1-\rho}+\left|\frac{\kappa}{H}-1\right|\right) \ell .
$$


Here $\ell=|\partial \Omega| /(4 \varepsilon), d(\ell)$ is introduced in (2.9), $\mathcal{E}, \mathbf{F}$ and $\psi_{\rho, \varepsilon}^{\text {bnd }}$ are the energy functional, the vector field and the test configuration introduced in (1.1), (1.2) and (4.11) respectively.

Proof. Let us introduce the vector field

$$
A(s, t)=\left(A_{1}(s, t), A_{2}(s, t)\right)=\left(-t+k(s) \frac{t^{2}}{2}, 0\right), \quad \forall(s, t) \in \mathbb{R}^{2},
$$

together with the two energies,

$$
A_{\varepsilon}=\int_{-\frac{|\partial \Omega|}{2}}^{0} \int_{0}^{t_{0}}\left([g(s, t)]^{-2}\left|\left(\partial_{s}-i \varepsilon^{-2} A_{1}\right) \phi_{\rho, \varepsilon}\right|^{2}+\left|\partial_{t} \phi_{\rho, \varepsilon}\right|^{2}-\kappa^{2}\left|\phi_{\rho, \varepsilon}\right|^{2}+\frac{\kappa^{2}}{2}\left|\phi_{\rho, \varepsilon}\right|^{4}\right) g(s, t) d t d s,
$$

and

$$
B_{\varepsilon}=\int_{0}^{-\frac{|\partial \Omega|}{2}} \int_{0}^{t_{0}}\left([g(s, t)]^{-2}\left|\left(\partial_{s}-i \varepsilon^{-2} A_{1}\right) \phi_{\rho, \varepsilon}\right|^{2}+\left|\partial_{t} \phi_{\rho, \varepsilon}\right|^{2}-\kappa^{2}\left|\phi_{\rho, \varepsilon}\right|^{2}+\frac{\kappa^{2}}{2}\left|\phi_{\rho, \varepsilon}\right|^{4}\right) g(s, t) d t d s .
$$

Here $g(s, t)=1-t k(s)$ and $t_{0}$ a sufficiently small constant as previously. Proposition 4.2, Lemma 4.3 and the definition of the function $\psi_{\rho, \varepsilon}^{\text {bnd }}$ all together give,

$$
\mathcal{E}\left(\psi_{\rho, \varepsilon}^{\mathrm{bnd}}, \mathbf{F} ; \Omega\right)=A_{\varepsilon}+B_{\varepsilon} .
$$

We claim that

and

$$
A_{\varepsilon} \leq d(\ell)+C\left(\varepsilon^{4-5 \rho}+\varepsilon^{\rho / 2}+\varepsilon^{1-\rho}+\left|\frac{\kappa}{H}-1\right|\right) \ell .
$$

$$
B_{\varepsilon} \leq d(\ell)+C\left(\varepsilon^{4-5 \rho}+\varepsilon^{\rho / 2}+\varepsilon^{1-\rho}+\left|\frac{\kappa}{H}-1\right|\right) \ell .
$$

Let us prove the upper bound for $A_{\varepsilon}$. The upper bound for $B_{\varepsilon}$ follows in the same way as for $A_{\varepsilon}$.

Define the rescaled variables $\sigma=\frac{s}{\varepsilon}+\ell, \tau=\frac{t}{\varepsilon}$ and the rescaled function

$$
u(\sigma, \tau)=\phi_{\rho, \varepsilon}(s, t), \quad \forall(\sigma, \tau) \in(-\ell, \ell) \times\left(0, \varepsilon^{\rho-1}\right),
$$

which is extended by zero for $(\sigma, \tau) \in(-\ell, \ell) \times\left[\varepsilon^{\rho-1}, \infty\right)$.

In the new scale, the expression for $A_{\varepsilon}$ becomes,

$$
A_{\varepsilon}=\int_{-\ell}^{\ell} \int_{0}^{\varepsilon^{\rho-1}}\left(\left[g_{\varepsilon}(\sigma, \tau)\right]^{-2}\left|\left(\partial_{\sigma}+i \tau-i \varepsilon a_{\varepsilon}\right) u\right|^{2}+\left|\partial_{\tau} u\right|^{2}-\frac{\kappa}{H}|u|^{2}+\frac{\kappa}{2 H}|u|^{4}\right) g_{\varepsilon}(\sigma, \tau) d \tau d \sigma .
$$

Here

$$
g_{\varepsilon}(\sigma, \tau)=1-\varepsilon k_{\varepsilon}(\sigma) \tau, \quad k_{\varepsilon}(\sigma)=k(\varepsilon(\sigma-\ell)), \quad a_{\varepsilon}(\sigma, \tau)=k_{\varepsilon}(\sigma) \frac{\tau^{2}}{2} .
$$

There exists a positive constant $C$ such that, for $\varepsilon$ sufficiently small, the following estimate holds,

$$
\frac{1}{2}<1-C \varepsilon^{\rho} \leq g_{\varepsilon}(\sigma, \tau) \leq 1+C \varepsilon^{\rho}, \quad \forall(\sigma, \tau) \in(-\ell, \ell) \times\left(0, \varepsilon^{\rho-1}\right) .
$$

Replacing $C$ by a larger constant, it holds for $\delta \in(0,1)$,

$$
\begin{aligned}
\int_{-\ell}^{\ell} \int_{0}^{\varepsilon^{\rho-1}}\left|\partial_{\tau} u\right|^{2} d \tau d \sigma & =\int_{-\ell}^{\ell} \int_{0}^{\varepsilon^{\rho-1}}\left|\chi_{\varepsilon}(\tau) \partial_{\tau} \phi_{\ell}+\phi_{\ell} \partial_{\tau} \chi_{\varepsilon}(\tau)\right|^{2} d \tau d \sigma \\
& \leq(1+\delta) \int_{-\ell}^{\ell} \int_{0}^{\infty}\left|\partial_{\tau} \phi_{\ell}\right|^{2} d \tau d \sigma+C \delta^{-1} \varepsilon^{2-2 \rho} \int_{-\ell}^{\ell} \int_{\frac{1}{2} \varepsilon^{\rho-1}}^{\varepsilon^{\rho-1}}\left|\phi_{\ell}\right|^{2} d \tau d \sigma .
\end{aligned}
$$

Here $\chi_{\varepsilon}(\tau)=\chi\left(\frac{\varepsilon \tau}{\varepsilon^{\rho}}\right)$ and $\phi_{\ell}$ a minimizer of the functional $\mathcal{E}_{\ell}$ in (2.6).

Using the decay of $\phi_{\ell}$ in Theorem 2.4. we get,

$$
\int_{-\ell}^{\ell} \int_{0}^{\varepsilon^{\rho-1}}\left|\partial_{\tau} u\right|^{2} d \tau d \sigma \leq(1+\delta) \int_{-\ell}^{\ell} \int_{0}^{\infty}\left|\partial_{\tau} \phi_{\ell}\right|^{2} d \tau d \sigma+C \delta^{-1} \varepsilon^{4-4 \rho}|\ln \varepsilon| \ell .
$$


In a similar fashion we get,

$$
\begin{aligned}
\int_{-\ell}^{\ell} \int_{0}^{\varepsilon^{\rho-1}} \mid & \left.\left(\partial_{\sigma}+i \tau-i \varepsilon a_{\varepsilon}\right) u\right|^{2} d \tau d \sigma \\
& \leq \int_{-\ell}^{\ell} \int_{0}^{\varepsilon^{\rho-1}}\left((1+\delta)\left|\left(\partial_{\sigma}+i \tau\right) \phi_{\ell}\right|^{2}+C \delta^{-1} \varepsilon^{2}\left|a_{\varepsilon} u\right|^{2}\right) d \tau d \sigma \\
& \leq(1+\delta) \int_{-\ell}^{\ell} \int_{0}^{\varepsilon^{\rho-1}}\left|\left(\partial_{\sigma}+i \tau\right) \phi_{\ell}\right|^{2} d \tau d \sigma+C \delta^{-1} \varepsilon^{2 \rho}|\ln \varepsilon| \ell
\end{aligned}
$$

Writing,

$$
\begin{aligned}
\frac{\kappa}{H} \int_{-\ell}^{\ell} \int_{0}^{\varepsilon^{\rho-1}}|u|^{2} d \tau d \sigma & =\int_{-\ell}^{\ell} \int_{0}^{\infty}\left|\phi_{\ell}\right|^{2} d \tau d \sigma+\int_{-\ell}^{\ell} \int_{\frac{1}{2} \varepsilon^{\rho-1}}^{\infty}\left(\left|\chi_{\varepsilon}\right|^{2}-1\right)\left|\phi_{\ell}\right|^{2} d \tau d \sigma \\
& +\left(\frac{\kappa}{H}-1\right) \int_{-\ell}^{\ell} \int_{0}^{\varepsilon^{\rho-1}}|u|^{2} d \tau d \sigma
\end{aligned}
$$

we get by the decay of $\phi_{\ell}$,

$$
\frac{\kappa}{H} \int_{-\ell}^{\ell} \int_{0}^{\varepsilon^{\rho-1}}|u|^{2} d \tau d \sigma \geq \int_{-\ell}^{\ell} \int_{0}^{\infty}\left|\phi_{\ell}\right|^{2} d \tau d \sigma-C \varepsilon^{1-\rho} \ell-C\left|\frac{\kappa}{H}-1\right| \ell .
$$

Similarly, we have the upper bound,

$$
\frac{\kappa}{H} \int_{-\ell}^{\ell} \int_{0}^{\varepsilon^{\rho-1}}|u|^{4} d \tau d \sigma \leq \int_{-\ell}^{\ell} \int_{0}^{\infty}\left|\phi_{\ell}\right|^{2} d \tau d \sigma+C\left|\frac{\kappa}{H}-1\right| \ell .
$$

Inserting the estimates (4.13)-(4.17) into (4.12) we get,

$$
A_{\varepsilon} \leq \mathcal{E}_{\ell}\left(\phi_{\ell}\right)+C\left(\delta^{-1}|\ln \varepsilon|\left(\varepsilon^{2 \rho}+\varepsilon^{4-4 \rho}\right)+\delta+\varepsilon^{\rho}+\varepsilon^{1-\rho}+\left|\frac{\kappa}{H}-1\right|\right) \ell .
$$

Here $\mathcal{E}_{\ell}$ is the functional in (2.6). Remembering that $\phi_{\ell}$ is a minimizer of $\mathcal{E}_{\ell}$, the definition of $d(\ell)$, and choosing $\delta=\varepsilon^{\rho / 2}$, we get the desired upper bound on $A_{\varepsilon}$.

4.2. Bulk configuration. In this section we construct a test configuration $\left(\psi^{\text {int }}, \mathbf{A}\right)$ using the limiting problem (2.21). Let us take $R>1$ (that will be chosen as a function of $\varepsilon$ such that $\varepsilon R \rightarrow 0$ as $\varepsilon \rightarrow 0$ ). Then, thanks to Proposition 2.10, the functional $F_{R}$ in (2.21) admits a minimizer $f_{R}$ in $L_{R}$, and we denote by,

$$
c(R)=F_{R}\left(f_{R}\right) .
$$

Recall the magnetic potential $\mathbf{A}_{0}$ introduced in (2.11). The configuration $\left(f_{R}, \mathbf{A}_{0}\right)$, defined initially on the unit lattice $K_{R}$, can be defined by periodicity in all $\mathbb{R}^{2}$.

Let us define now the following test configuration in $\Omega$,

$$
\psi_{\rho, R, \varepsilon}^{\mathrm{int}}(x)=\kappa^{-1 / 4} h\left(R^{\rho} \frac{\operatorname{dist}(x, \partial \Omega)}{2}\right) f_{R}\left(\frac{x}{\varepsilon}\right), \quad \forall x \in \Omega .
$$

Here $\rho>0$ is to be fixed later, and $h \in C^{\infty}(\mathbb{R})$ is a cut-off function such that

$$
0 \leq h \leq 1 \text { in } \mathbb{R}, \quad h=1 \text { in }[1, \infty), \quad \operatorname{supp} h \subset(0, \infty) .
$$

Notice that we can cover $\Omega$ by $N_{\varepsilon}$ squares of the lattice $\varepsilon R(\mathbb{Z} \oplus i \mathbb{Z})$, where $N_{\varepsilon}$ satisfies,

$$
\lim _{\varepsilon \rightarrow 0}\left[N_{\varepsilon} \times\left(\varepsilon^{2} R^{2}\right)\right]=|\Omega| .
$$

The next lemma gives an estimate of the energy of the test configuration (4.18). 
Lemma 4.5. Let $\rho \in(0,1)$ be a given constant. Assume that $R=R(\varepsilon)$ is a function satisfying $\left|K_{R}\right| \in 2 \pi \mathbb{N}$ and $1 \ll R \ll \varepsilon^{-\frac{1}{1+\rho}}$ as $\varepsilon \rightarrow 0$. There exist positive constants $\varepsilon_{0}$, $C$, and a function

$$
\mathbb{R}_{+} \ni t \mapsto \delta(t) \in \mathbb{R}_{+}, \quad \lim _{t \rightarrow \infty} \delta(t)=0,
$$

such that, for all $\varepsilon \in\left(0, \varepsilon_{0}\right], \mu>0$, and if the magnetic field satisfies,

$$
H=\kappa-\mu \sqrt{\kappa},
$$

then we have the following estimate,

$$
\mathcal{E}\left(\mu^{1 / 2} \psi_{\rho, R, \varepsilon}^{\text {int }}, \mathbf{A}_{0}\right) \leq \mu^{2} \kappa|\Omega| c(R)+C \mu\left(\kappa^{-1 / 2} R^{\rho}+\kappa^{3 / 2} R^{-\rho}\right)+\mu^{2} \kappa \delta(\kappa) .
$$

Here $c(R)$ is introduced in (2.23).

Proof. Let us denote by $\widetilde{f}_{R}(x)=f_{R}(x / \varepsilon)$ and $h_{R}(x)=h\left(R^{\rho} \frac{\operatorname{dist}(x, \partial \Omega)}{2}\right)$. Notice that we have the following localization formula,

$$
\begin{aligned}
\int_{\Omega}\left|\left(\nabla-i \varepsilon^{-2} \mathbf{A}_{0}\right) \psi_{\rho, R, \varepsilon}^{\text {int }}\right|^{2} d x=\kappa^{-1 / 2} \Re\left\langle-h_{R}^{2}\left(\nabla-i \varepsilon^{-2} \mathbf{A}_{0}\right)^{2} \widetilde{f}_{R}, \widetilde{f}_{R}\right\rangle_{L^{2}(\Omega)} & \\
& +\left.\left.\kappa^{-1 / 2} \int_{\Omega}|| \nabla h_{R}\right|^{2} \widetilde{f}_{R}\right|^{2} d x .
\end{aligned}
$$

From the definition of $\widetilde{f}_{R}$ and $\mathbf{A}_{0}$, and a simple scaling, we may check that

$$
-\left(\nabla-i \varepsilon^{-2} \mathbf{A}_{0}\right)^{2} \widetilde{f}_{R}=\varepsilon^{-2} \widetilde{f}_{R}=\kappa H \widetilde{f}_{R} .
$$

Therefore, (4.20) becomes,

$$
\begin{aligned}
& \kappa^{1 / 2} \int_{\Omega}\left|\left(\nabla-i \varepsilon^{-2} \mathbf{A}_{0}\right) \psi_{\rho, R, \varepsilon}^{\text {int }}\right|^{2} d x \\
& \quad=\kappa H \int_{\Omega}\left|h_{R} \widetilde{f}_{R}\right|^{2} d x+\int_{\Omega}\left|\nabla h_{R}\right|^{2}\left|\widetilde{f}_{R}\right|^{2} d x \\
& \quad \leq \kappa H \int_{\Omega}\left|\widetilde{f}_{R}\right|^{2} d x+2\left\|h^{\prime}\right\|_{L^{\infty}(\mathbb{R})}^{2} R^{2 \rho} \int_{\left\{2 R^{-\rho} \leq \operatorname{dist}(x, \partial \Omega) \leq 4 R^{-\rho}\right\}}\left|\widetilde{f}_{R}\right|^{2} d x
\end{aligned}
$$

Let us estimate the last term in (4.21), which is in fact a remainder term. Recall that $\tilde{f}_{R}$ is periodic with respect to the lattice $\varepsilon R(\mathbb{Z} \oplus i \mathbb{Z})$. Using the condition $R \ll \varepsilon^{-\frac{1}{1+\rho}}$, we cover $\left\{x \in \Omega: 2 R^{-\rho} \leq \operatorname{dist}(x, \partial \Omega) \leq 4 R^{-\rho}\right\}$ by $N_{\varepsilon}^{\prime}$ squares of the lattice $\varepsilon R(\mathbb{Z} \oplus i \mathbb{Z})$, with $N_{\varepsilon}^{\prime} \leq C \frac{R^{-\rho}}{\varepsilon^{2} R^{2}}$. Therefore,

$$
\int_{\left\{2 R^{-\rho} \leq \operatorname{dist}(x, \partial \Omega) \leq 4 R^{-\rho}\right\}}\left|\widetilde{f}_{R}\right|^{2} d x \leq C \frac{R^{-\rho}}{\varepsilon^{2} R^{2}} \int_{K_{\varepsilon R}}\left|\widetilde{f}_{R}(x)\right|^{2} d x=C R^{-2-\rho} \int_{K_{R}}\left|f_{R}(x)\right|^{2} d x .
$$

Invoking the estimate of Proposition 2.10, we get,

$$
\int_{\left\{2 R^{-\rho} \leq \operatorname{dist}(x, \partial \Omega) \leq 4 R^{-\rho}\right\}}\left|\widetilde{f}_{R}\right|^{2} d x \leq C R^{-\rho} .
$$

Next we estimate $\left\|h_{R} \widetilde{f}_{R}\right\|_{L^{2}(\Omega)}$ from below. Notice that, since $0 \leq h_{R} \leq 1$ and $1-h_{R}$ is supported in a thin neighborhood near the boundary, we have,

$$
\begin{aligned}
\int_{\Omega}\left|h_{R}(x) \tilde{f}_{R}(x)\right|^{2} d x & =\int_{\Omega}\left|\widetilde{f}_{R}(x)\right|^{2} d x-\int_{\Omega}\left(1-h_{R}^{2}(x)\right)\left|\widetilde{f}_{R}(x)\right|^{2} d x \\
& \geq \int_{\Omega}\left|\widetilde{f}_{R}(x)\right|^{2} d x-\int_{\left\{x \in \Omega: \operatorname{dist}(x, \partial \Omega) \leq 2 R^{-\rho}\right\}}\left|\widetilde{f}_{R}(x)\right|^{2} d x .
\end{aligned}
$$

Similarly as for (4.22), the estimate of Proposition 2.10 gives

$$
\int_{\left\{x \in \Omega: \operatorname{dist}(x, \partial \Omega) \leq 2 R^{-\rho}\right\}}\left|\widetilde{f}_{R}(x)\right|^{2} d x \leq C R^{-\rho},
$$


and therefore,

$$
\int_{\Omega}\left|h_{R}(x) \widetilde{f}_{R}(x)\right|^{2} d x \geq \int_{\Omega}\left|\widetilde{f}_{R}(x)\right|^{2} d x-C R^{-\rho} .
$$

Collecting (4.21), (4.22) and (4.23), and remembering that curl $\mathbf{A}_{0}=1$ by construction, we get finally,

$$
\begin{aligned}
\mathcal{E}\left(\mu^{1 / 2} \psi_{\rho, R, \varepsilon}^{\text {int }}, \mathbf{A}_{0}\right) & \leq \sqrt{\kappa}(H-\kappa) \mu \int_{\Omega}\left|\widetilde{f}_{R}\right|^{2} d x+\frac{\kappa \mu^{2}}{2} \int_{\Omega}\left|\widetilde{f}_{R}\right|^{4} d x+C \mu\left(\kappa^{-1 / 2} R^{\rho}+\kappa^{3 / 2} R^{-\rho}\right) \\
& =\mu^{2} \kappa \int_{\Omega}\left(\frac{1}{2}\left|\widetilde{f}_{R}\right|^{4}-\left|\widetilde{f}_{R}\right|^{2}\right) d x+C \mu\left(\kappa^{-1 / 2} R^{\rho}+\kappa^{3 / 2} R^{-\rho}\right) .
\end{aligned}
$$

We have to estimate the integral in (4.24). Toward that end, we define two sets $\underline{\mathcal{I}}$ and $\overline{\mathcal{I}}$ as follows. A square $K$ of the lattice $\varepsilon R(\mathbb{Z} \oplus i \mathbb{Z})$ belongs to $\mathcal{I}$ if $K \subset \Omega$; if $K \cap \Omega \neq \emptyset$ then $K$ belongs to $\overline{\mathcal{I}}$. Let us introduce the two integers,

$$
\underline{N}_{\varepsilon}=\operatorname{Card}(\underline{\mathcal{I}}), \quad \bar{N}_{\varepsilon}=\operatorname{Card}(\overline{\mathcal{I}}) .
$$

The formula in (4.19) still holds for both $\underline{N}_{\varepsilon}$ and $\bar{N}_{\varepsilon}$. Furthermore, by periodicity of $\left|\tilde{f}_{R}\right|$, we get,

$$
\begin{aligned}
\int_{\Omega} \frac{1}{2}\left|\widetilde{f}_{R}\right|^{4}-\left|\widetilde{f}_{R}\right|^{2} & \leq \bar{N}_{\varepsilon} \frac{1}{2} \int_{K_{\varepsilon R}}\left|\widetilde{f}_{R}\right|^{4}-\underline{N}_{\varepsilon} \int_{K_{\varepsilon R}}\left|\widetilde{f}_{R}\right|^{2} \\
& =\frac{|\Omega|}{\left|K_{R}\right|}\left(\frac{1+o(1)}{2} \int_{K_{R}}\left|f_{R}\right|^{4}-(1+o(1)) \int_{K_{R}}\left|f_{R}\right|^{2}\right) \\
& =|\Omega| c(R)+o(1) \quad \text { as } \varepsilon \rightarrow 0 .
\end{aligned}
$$

In the last step above we used the definition of $f_{R}$ and Proposition 2.10

Upon substitution in (4.24), we get,

$$
\begin{aligned}
& \mathcal{E}\left(\mu^{1 / 2} \psi_{\rho, R, \varepsilon}^{\text {int }}, \mathbf{A}_{0}\right) \\
& \quad \leq \mu^{2} \kappa(|\Omega| c(R) 1+o(1))+C \mu\left(\kappa^{-1 / 2} R^{\rho}+\kappa^{3 / 2} R^{-\rho}\right) \quad \text { as } \varepsilon \rightarrow 0,
\end{aligned}
$$

which is what we wanted to prove.

4.3. Proof of Theorem 4.1. Let $\mu=\mu(\kappa)$ be given by $H=\kappa-\mu(\kappa) \sqrt{\kappa}$. Let us define the following test function,

$$
\psi(x)=\psi_{\rho, \varepsilon}^{\mathrm{bnd}}(x)+[\mu]_{+}^{1 / 2} e^{-i \kappa H \varphi_{0}} \psi_{\rho, R, \varepsilon}^{\mathrm{bnd}}(x),
$$

and evaluate the energy $\mathcal{E}(\psi, \mathbf{F} ; \Omega)$. Here $\psi_{\rho, \varepsilon}^{\text {bnd }}$ is introduced in (4.11), $\psi_{\rho, R, \varepsilon}^{\text {int }}$ in (4.18), $\mathbf{F}$ the vector field introduced in (1.2), and the function $\varphi_{0}$ is to be specified later. Since $1 \ll R \ll \varepsilon^{-\frac{1}{1+\rho}}$, we see that $\psi_{\rho, \varepsilon}^{\text {bnd }}$ and $\psi_{\rho, R, \varepsilon}^{\text {int }}$ have disjoint supports, hence

$$
\mathcal{E}(\psi, \mathbf{F} ; \Omega)=\mathcal{E}\left(\psi_{\rho, \varepsilon}^{\mathrm{bnd}}, \mathbf{F} ; \Omega\right)+\mathcal{E}\left([\mu]_{+}^{1 / 2} e^{-i \kappa H \varphi_{0}} \psi_{\rho, R, \varepsilon}^{\text {int }}, \mathbf{F} ; \Omega\right) .
$$

We impose the condition $\rho \in\left(0, \frac{1}{2}\right)$. Then, thanks to Lemma 4.4, we get the following upper bound,

$$
\mathcal{E}\left(\psi_{\rho, \varepsilon}^{\mathrm{bnd}}, \mathbf{F} ; \Omega\right) \leq-E_{1}|\partial \Omega| \kappa+o(\kappa) .
$$

So we need to estimate the term $E\left([\mu]_{+}^{1 / 2} e^{-i \kappa H \varphi_{0}} \psi_{\rho, R, \varepsilon}^{\text {int }}, \mathbf{F} ; \Omega\right)$.

Recall the vector potential $\mathbf{A}_{0}$ introduced in (2.11). Notice that $\operatorname{curl} \mathbf{F}=\operatorname{curl} \mathbf{A}_{0}=1$. So, defining the function $\varphi_{0}$ by

$$
-\Delta \varphi_{0}=\operatorname{div} \mathbf{A}_{0}=0 \quad \text { in } \Omega, \quad \nu \cdot \nabla \varphi_{0}=\nu \cdot \mathbf{A}_{0} \quad \text { on } \partial \Omega,
$$


we get $\mathbf{F}=\mathbf{A}_{0}-\nabla \varphi_{0}$. Therefore,

$$
\mathcal{E}\left([\mu]_{+}^{1 / 2} e^{-i \varphi_{0}} \psi_{\rho, R, \varepsilon}^{\text {int }}, \mathbf{F} ; \Omega\right)=\mathcal{E}\left([\mu]_{+}^{1 / 2} \psi_{\rho, R, \varepsilon}^{\text {int }}, \mathbf{A}_{0} ; \Omega\right) .
$$

Thanks to Lemma 4.5 and the definition of $E_{2}$ in (2.24), we get,

$$
\mathcal{E}\left([\mu]_{+}^{1 / 2} \psi_{\rho, R, \varepsilon}^{\mathrm{int}}, \mathbf{A}_{0}\right) \leq-[\mu]_{+}^{2} E_{2}|\Omega| \kappa+C[\mu]_{+}\left(\kappa^{-1 / 2} R^{\rho}+\kappa^{3 / 2} R^{-\rho}\right)+o\left([\mu]_{+}^{2} \kappa\right) .
$$

Remembering the condition $\rho \in\left(0, \frac{1}{2}\right)$ and taking $R=2 \pi \sqrt{\left[\kappa^{\rho}\right]+1}$, we get,

$$
\mathcal{E}\left([\mu]_{+}^{1 / 2} \psi_{\rho, R, \varepsilon}^{\mathrm{int}}, \mathbf{A}_{0}\right) \leq-E_{2}|\Omega|[\mu]_{+}^{2} \kappa+o\left(\max \left(1,[\mu]_{+}^{2}\right) \kappa\right) .
$$

This finishes the proof of Theorem 4.1 .

\section{LOWER BOUND OF THE ENERGY}

Let us pick a minimizer $(\psi, \mathbf{A})$ of the Ginzburg-Landau energy (1.1). Our aim in this section is to give a lower bound of the energy $\mathcal{E}(\psi, \mathbf{A} ; \Omega)$. We recall the convention that an open subset $D \subset \Omega$ is smooth if there exists an open and smooth set $\widetilde{D} \subset \mathbb{R}^{2}$ such that $D=\widetilde{D} \cap \Omega$. For all $a>0$, we associate with a subset $D \subset \Omega$ the following subset of $\Omega$,

$$
D_{a}=\{x \in \Omega: \operatorname{dist}(x, D) \leq a\} .
$$

We will prove the following theorem.

Theorem 5.1. Assume the magnetic field satisfies $H=\kappa-\mu(\kappa) \sqrt{\kappa}$ with $\lim _{\kappa \rightarrow \infty} \frac{\mu(\kappa)}{\sqrt{\kappa}}=0$ and $\liminf _{\kappa \rightarrow \infty} \mu(\kappa)>-\infty$. Let $D \subset \Omega$ be smooth, open, and $\mathbb{R}_{+} \ni \kappa \mapsto a(\kappa) \in \mathbb{R}_{+}$a function satisfying $\lim _{\kappa \rightarrow \infty} a(\kappa)=0$.

Then, for any minimizer $(\psi, \mathbf{A})$ of the energy $\mathcal{E}$ in (1.1) and any continuous function $h \in C(\Omega)$ satisfying $\|h\|_{L^{\infty}(\Omega)} \leq 1$ and $\operatorname{supp} h \subset \bar{D}_{a}$, the following asymptotic lower bound holds,

$$
\mathcal{E}(h \psi, \mathbf{A} ; \Omega) \geq-E_{1}|\bar{D} \cap \partial \Omega| \kappa-E_{2}|D|[\mu(\kappa)]_{+}^{2} \kappa+o\left(\max \left(1,[\mu(\kappa)]_{+}^{2}\right) \kappa\right), \quad \text { as } \kappa \rightarrow \infty .
$$

Here $E_{1}$ and $E_{2}$ are the constants introduced in (2.10) and 2.24) respectively.

Recall the ground state energy $C_{0}(\kappa, H)$ introduced in (1.3). As corollary from Theorem 5.1, we get an asymptotic lower bound for $C_{0}(\kappa, H)$.

Corollary 5.2. Assume $H=\kappa-\mu(\kappa) \sqrt{\kappa}$ and $\frac{\mu(\kappa)}{\sqrt{\kappa}} \rightarrow 0$ as $\kappa \rightarrow \infty$. The following lower bound holds for the ground state energy in (1.3),

$$
C_{0}(\kappa, H) \geq-E_{1}|\partial \Omega| \kappa-E_{2}|\Omega|[\mu(\kappa)]_{+}^{2} \kappa+o\left(\max \left(1,[\mu(\kappa)]_{+}^{2}\right) \kappa\right), \quad \text { as } \kappa \rightarrow \infty .
$$

Here $E_{1}>0$ and $E_{2}>0$ are the constants introduced in (2.10) and 2.24) respectively.

Proof. Assume the conclusion of the corollary were false and let $\left\{\kappa_{n}\right\}$ be a sequence such that $\kappa_{n} \rightarrow \infty$ and $\mu\left(\kappa_{n}\right) \rightarrow \mu_{0}$ as $n \rightarrow \infty$, with $\mu_{0} \in \mathbb{R} \cup\{ \pm \infty\}$.

If $\mu_{0}=-\infty$, we apply Theorem 1.1 (which is proved by Pan in [12]), with $\kappa=\kappa_{n}$ and $D=\Omega$. Otherwise, if $\mu_{0} \in \mathbb{R} \cup\{\infty\}$, we apply Theorem [5.1] with $D=\Omega, h \equiv 1$ and $\kappa=\kappa_{n}$. In both cases we get a contradiction to the assumption that the conclusion of the corollary were fasle.

The general strategy for proving Theorem 5.1 is the following. Using a partition of unity we may split the energy into a boundary component and a bulk component. To control the boundary component of the energy we follow essentially the argument of Pan [12]. However, the control of the bulk component of the energy is novel. Finally, we make use of the a priori estimates recalled in Lemma 2.2 and the improved estimate in Corollary 3.1 in order to control the errors resulting from the approximations (this is one additional key point that replaces the implementation of the exponential decay of $\psi$ in Pan's argument [12]). 
In what follows, we consider a domain $D=\widetilde{D} \cap \Omega$, with $\widetilde{D}$ a smooth and open domain in $\mathbb{R}^{2}$. We assume for simplicity the following condition on $D$,

$$
\bar{D} \text { is connected, } \quad \bar{D} \cap \partial \Omega=\partial \Omega, \quad|D| \neq|\Omega| .
$$

In general, $\bar{D}$ consists of a finite number of connected components. By working with each component separately and adding up the corresponding lower bounds, one can reduce to the connected case. Furthermore, $\bar{D} \cap \partial \Omega$ consists of a finite union of closed curves (possibly of zero length). A simple modification of the argument below will handle this case as well, so we therefore only treat domains $D$ satisfying the condition in (5.4).

5.1. Splitting of the energy. Let us consider a parameter $\eta=\eta(\kappa)>0$ such that $\eta \rightarrow 0$ as $\kappa \rightarrow \infty$. We will take $\eta$ in the following form

$$
\eta=\kappa^{-\rho}, \quad \rho \in\left(\frac{1}{4}, 1\right),
$$

and we will fix a choice of $\rho$ at the end of the proof.

Let us also consider a partition of unity

$$
\chi_{1}^{2}+\chi_{2}^{2}=1 \quad \text { in } \mathbb{R}, \quad \operatorname{supp} \chi_{1} \subset(-\infty, 2], \quad \operatorname{supp} \chi_{2} \subset[1, \infty) .
$$

We define,

$$
\chi_{1, \eta}(x)=\chi_{1}\left(\frac{\operatorname{dist}(x, \partial \Omega)}{\eta}\right), \quad \chi_{2, \eta}(x)=\chi_{2}\left(\frac{\operatorname{dist}(x, \partial \Omega)}{\eta}\right), \quad \forall x \in \Omega .
$$

Then we get the following localization formula,

$$
\int_{\Omega}|(\nabla-i \kappa H \mathbf{A}) h \psi|^{2} d x=\sum_{j=1}^{2} \int_{\Omega}\left(\left|(\nabla-i \kappa H \mathbf{A}) \chi_{j, \eta} h \psi\right|^{2}-|| \nabla \chi_{j, \eta}|h \psi|^{2}\right) d x .
$$

Defining the reduced energy,

$$
\mathcal{E}_{0}(\psi, \mathbf{A} ; \Omega)=\int_{\Omega}\left(|(\nabla-i \kappa H \mathbf{A}) \psi|^{2}-\kappa^{2}|\psi|^{2}+\frac{\kappa^{2}}{2}|\psi|^{4}\right) d x,
$$

we then get in light of (5.7),

$$
\mathcal{E}(h \psi, \mathbf{A} ; \Omega) \geq \sum_{j=1}^{2} \mathcal{E}_{0}\left(\chi_{j, \eta} h \psi, \mathbf{A} ; \Omega\right)-\mathcal{R}(h \psi, \mathbf{A}),
$$

where

$$
\mathcal{R}(h \psi, \mathbf{A})=\sum_{j=1}^{2} \int_{\Omega}|| \nabla \chi_{j, \eta}|h \psi|^{2} d x .
$$

This last term corresponds to an error which we will estimate using Theorem 1.4 Also, recall the assumption that $|h| \leq 1$ in $\Omega$. In this way we get,

$$
\mathcal{R}(h \psi, \mathbf{A}) \leq\left\|\nabla \chi_{j, \eta}\right\|_{L^{\infty}(\Omega)}^{2}\|\psi\|_{L^{\infty}\left(\omega_{\kappa}\right)}^{2} \int_{\{\eta \leq \operatorname{dist}(x, \partial \Omega) \leq 2 \eta\}} d x \leq C .
$$

Here $\omega_{\kappa}=\left\{x \in \Omega: \operatorname{dist}(x, \partial \Omega) \geq \kappa^{-\rho}\right\}$ and

$$
\zeta=\zeta(\kappa)=\max \left\{\left|1-\frac{\kappa}{H}\right|^{1 / 2}, \kappa^{-1 / 4}\right\} .
$$

Upon substitution into (5.9) we get,

$$
\mathcal{E}(h \psi, \mathbf{A} ; \Omega) \geq \sum_{j=1}^{2} \mathcal{E}_{0}\left(\chi_{j, \eta} h \psi, \mathbf{A} ; \Omega\right)-\frac{C}{\eta} \zeta^{2} .
$$

We proceed to estimate separately the terms $\mathcal{E}_{0}\left(\chi_{1, \eta} h \psi, \mathbf{A} ; \Omega\right)$ and $\mathcal{E}_{0}\left(\chi_{2, \eta} h \psi, \mathbf{A} ; \Omega\right)$. 
5.2. Estimating the boundary energy. Let us now introduce a further partition of unity, $h_{1}^{2}+h_{2}^{2}=1 \quad$ in $\mathbb{R}, \quad \operatorname{supp} h_{1} \subset(-\infty, 1), \quad \operatorname{supp} h_{2} \subset(-1, \infty)$,

and such that

$$
h_{1}=1 \quad \text { in }(-\infty,-1], \quad h_{2}=1 \text { in }[1, \infty) .
$$

Let $s_{0}=|\partial \Omega| / 4$. Recall the coordinate transformation $\Phi$ in (4.2) valid in the neighborhood $\Omega\left(t_{0}\right)$ of $\partial \Omega$. By defining,

$$
h_{1, \eta}(x)=h_{1}\left(\frac{|s(x)|-s_{0}}{\eta}\right), \quad h_{2, \eta}(x)=h_{2}\left(\frac{|s(x)|-s_{0}}{\eta}\right) \quad \forall x \in \Omega(\eta),
$$

we get a partition of unity in $\Omega(\eta)$. Using the localization formula, the energy splits one more time as follows,

$$
\mathcal{E}_{0}\left(\chi_{j, \eta} h \psi, \mathbf{A} ; \Omega\right)=\sum_{j=1}^{2}\left(\mathcal{E}_{0}\left(\psi_{j, \eta}, \mathbf{A} ; \Omega\right)-\int_{\Omega}|| \nabla h_{j, \eta}\left|\chi_{1, \eta} h \psi\right|^{2} d x\right),
$$

where

$$
\psi_{j, \eta}(x)=h_{j, \eta}(x) \chi_{1, \eta}(x) h(x) \psi(x), \quad \forall x \in \Omega(\eta) .
$$

Noticing that the supports of the functions $\left|\nabla h_{j, \eta}\right| \chi_{1, \eta} \psi, j=1,2$, are contained in $\{x \in$ $\left.\Omega: \operatorname{dist}(x, \partial \Omega) \leq 2 \eta, 0 \leq|| s(x)\left|-s_{0}\right| \leq \eta\right\}$, we obtain for a possibly new constant $C$,

$$
\sum_{j=1}^{2} \int_{\Omega}|| \nabla h_{j, \eta}\left|\chi_{1, \eta} h \psi\right|^{2} d x \leq C \eta .
$$

Substituting this into (5.12), we obtain,

$$
\mathcal{E}_{0}\left(\chi_{j, \eta} h \psi, \mathbf{A} ; \Omega\right) \geq \sum_{j=1}^{2} \mathcal{E}_{0}\left(\psi_{j, \eta}, \mathbf{A} ; \Omega\right)-C .
$$

Let us now bound the term $\mathcal{E}_{0}\left(\psi_{1, \eta}, \mathbf{A} ; \Omega\right)$ from below. Since curl $A=1$ on $\partial \Omega$, Lemma F.1.1 of [6] yields that up to a gauge transformation, we may write in $(s, t)$-coordinates,

$$
\widetilde{\mathbf{A}}(s, t)=\left(-t+\frac{t^{2}}{2} k(s)+t^{2} b(s, t), 0\right) \quad \text { in } \operatorname{supp} h_{1, \eta},
$$

where the function $b$ satisfies

$$
|b(s, t)| \leq\|\operatorname{curl} \mathbf{A}-1\|_{C^{1}(\Omega)} \leq C \kappa^{-1}, \quad \forall(s, t) \in \operatorname{supp} h_{1, \eta},
$$

and where we used Lemma 2.2 in the last step. We also remind the reader that $\widetilde{\mathbf{A}}=U_{\Phi} \mathbf{A}$ is the vector field associated to $\mathbf{A}$ by the coordinate transformation $\Phi$.

It is more convenient in this part to introduce the parameters,

$$
\varepsilon=\frac{1}{\sqrt{\kappa H}}, \quad \gamma=\frac{\kappa}{H}-1=\mu(\kappa) \frac{\sqrt{\kappa}}{H}=\mathcal{O}(\mu \sqrt{\varepsilon}) .
$$

Then, applying the change of variables formula in Proposition 4.2, we get,

$$
\mathcal{E}_{0}\left(\psi_{1, \eta}, \mathbf{A} ; \Omega\right)=\mathcal{J}_{\varepsilon}\left(\widetilde{\psi}_{1, \eta}\right)
$$

where

$$
\begin{aligned}
\mathcal{J}_{\varepsilon}\left(\widetilde{\psi}_{1, \eta}\right)=\int_{-\frac{|\partial \Omega|}{2}}^{\frac{|\partial \Omega|}{2}} \int_{0}^{t_{0}}[ & (1-t k(s))^{-2}\left|\left(\partial_{s}+\frac{i}{\varepsilon^{2}}(t+\widetilde{b}(s, t))\right) \widetilde{\psi}_{1, \eta}\right|^{2} \\
& \left.+\left|\partial_{t} \widetilde{\psi}_{1, \eta}\right|^{2}-\frac{1+\gamma}{\varepsilon^{2}}\left|\widetilde{\psi}_{1, \eta}\right|^{2}+\frac{1+\gamma}{2 \varepsilon^{2}}\left|\widetilde{\psi}_{1, \eta}\right|^{4}\right](1-t k(s)) d s d t,
\end{aligned}
$$


and

$$
\widetilde{b}(s, t)=-t^{2}\left(\frac{k(s)}{2}+b(s, t)\right)=\mathcal{O}\left(t^{2}\right) .
$$

Let us introduce another parameter $\delta=\delta(\varepsilon) \in(0,1)$ to be fixed later. Applying a CauchySchwarz inequality, we get,

$$
\left|\left(\partial_{s}+\frac{i}{\varepsilon^{2}}(t+\widetilde{b}(s, t))\right) \widetilde{\psi}_{1, \eta}\right|^{2} \geq(1-\delta)\left|\left(\partial_{s}+\frac{i}{\varepsilon^{2}} t\right) \widetilde{\psi}_{1, \eta}\right|^{2}-\left.\delta^{-1} \frac{1}{\varepsilon^{4}}|\widetilde{b}(s, t)|^{2} \widetilde{\psi}_{1, \eta}\right|^{2} .
$$

Substituting the above estimate into (5.16), we get,

$$
\mathcal{J}_{\varepsilon}\left(\widetilde{\psi}_{1, \eta}\right) \geq(1-\delta) \mathcal{Q}_{\varepsilon}\left(\widetilde{\psi}_{1, \eta}\right)-C \mathcal{R}_{\text {bnd }}\left(\widetilde{\psi}_{1, \eta}\right)
$$

where

$$
\mathcal{Q}_{\varepsilon}\left(\widetilde{\psi}_{1, \eta}\right)=\int_{-\frac{|\partial \Omega|}{2}}^{\frac{|\partial \Omega|}{2}} \int_{0}^{t_{0}}\left(\left|\left(\partial_{s}+\frac{i}{\varepsilon^{2}} t\right) \widetilde{\psi}_{1, \eta}\right|^{2}+\left|\partial_{t} \widetilde{\psi}_{1, \eta}\right|^{2}-\frac{1}{\varepsilon^{2}}\left|\widetilde{\psi}_{1, \eta}\right|^{2}+\frac{1}{2 \varepsilon^{2}}\left|\widetilde{\psi}_{1, \eta}\right|^{4}\right) d s d t
$$

and

$$
\begin{aligned}
& \mathcal{R}_{\text {bnd }}\left(\widetilde{\psi}_{1, \eta}\right)=\frac{1}{\varepsilon^{2}} \int\left[|\gamma|+t+\frac{\delta^{-1}}{\varepsilon^{2}} t^{4}\right]\left|\widetilde{\psi}_{1, \eta}\right|^{2} d s d t+ \\
& \qquad t\left(\left|\left(\partial_{s}+\frac{i}{\varepsilon^{2}}(t+\widetilde{b}(s, t))\right) \widetilde{\psi}_{1, \eta}\right|^{2}+\left|\partial_{t} \widetilde{\psi}_{1, \eta}\right|^{2}\right) d s d t .
\end{aligned}
$$

From the definition of $\psi_{1, \eta}$, we know that

$$
\operatorname{supp} \psi_{1, \eta} \subset\left\{x \in \Omega \quad \operatorname{dist}(x, \partial \Omega) \leq \eta,|s(x)| \leq \frac{|\partial \Omega|}{4}+\eta\right\}, \quad\left|\psi_{1, \eta}(x)\right| \leq|\psi(x)| \leq 1 .
$$

With this point on the one hand, and (2.3) on the other hand, we deduce that,

$$
\mathcal{R}_{\text {bnd }}\left(\widetilde{\psi}_{1, \eta}\right) \leq \frac{C}{\varepsilon^{2}}\left(\eta|\gamma|+\eta^{2}+\frac{\delta^{-1}}{\varepsilon^{2}} \eta^{5}\right) .
$$

Let us define the re-scaled function,

$$
g_{\eta}(\sigma, \tau)= \begin{cases}\widetilde{\psi}_{1, \eta}(\varepsilon \sigma, \varepsilon \tau) & \text { if }(\sigma, \tau) \in(-\ell, \ell) \times\left(0, \eta \varepsilon^{-1}\right), \\ 0 & \text { otherwise }\end{cases}
$$

where

In the new scale, we may write,

$$
\ell=\frac{|\partial \Omega|}{4 \varepsilon}+\frac{\eta}{\varepsilon}
$$

$$
\mathcal{Q}_{\varepsilon}\left(\widetilde{\psi}_{1, \eta}\right)=\mathcal{E}_{\ell}\left(g_{\eta}\right),
$$

where $\mathcal{E}_{\ell}$ is the functional introduced in (2.6). Invoking Theorem 2.4, we get a new constant $M>0$ such that,

$$
\mathcal{Q}_{\varepsilon}\left(\widetilde{\psi}_{1, \eta}\right) \geq d(\ell) \geq-E_{1}\left(\frac{|\partial \Omega|}{2 \varepsilon}+2 \frac{\eta}{\varepsilon}\right)-M .
$$

Summing up the estimates in (5.15), (5.18), (5.19) and (5.20), we get finally,

$$
\mathcal{E}_{0}\left(\psi_{1, \eta}, \mathbf{A} ; \Omega\right) \geq-\left(E_{1} \frac{|\partial \Omega|}{2 \varepsilon}+C \frac{\eta}{\varepsilon}+M\right)(1-\delta)-\frac{C}{\varepsilon^{2}}\left(\eta^{2}+\eta|\gamma|+\frac{\delta^{-1}}{\varepsilon^{2}} \eta^{5}\right) .
$$

In a similar fashion, we establish that,

$$
\mathcal{E}_{0}\left(\psi_{2, \eta}, \mathbf{A} ; \Omega\right) \geq-\left(E_{1} \frac{|\partial \Omega|}{2 \varepsilon}+C \frac{\eta}{\varepsilon}+M\right)(1-\delta)-\frac{C}{\varepsilon^{2}}\left(\eta^{2}+\eta \gamma+\frac{\delta^{-1}}{\varepsilon^{2}} \eta^{5}\right) .
$$


Invoking (5.14), and recalling the definition of $\gamma$ and $\varepsilon=\kappa^{-1}(1+o(1))$, we get,

$$
\mathcal{E}_{0}\left(\chi_{1, \eta} h \psi, \mathbf{A} ; \Omega\right) \geq-E_{1}|\partial \Omega| \kappa-C \kappa^{2}\left(\eta^{2}+\frac{\eta|\mu(\kappa)|}{\sqrt{\kappa}}+\delta^{-1} \kappa^{2} \eta^{5}\right)-C(\delta+\eta) \kappa .
$$

5.3. Estimating the bulk energy. We recall that, for a given $R>0$, we denote by $K_{R}$ the unit square of the lattice $R(\mathbb{Z} \oplus i \mathbb{Z})$.

For $x \in \mathbb{R}^{2}$ and $R>0$, we denote by $K_{R}(x)$ a square of center $x$ and side length $R$,

$$
K_{R}(x)=\left(x_{1}-\frac{R}{2}, x_{1}+\frac{R}{2}\right) \times\left(x_{2}-\frac{R}{2}, x_{2}+\frac{R}{2}\right), \quad \forall x=\left(x_{1}, x_{2}\right) \in \mathbb{R}^{2}, R>0 .
$$

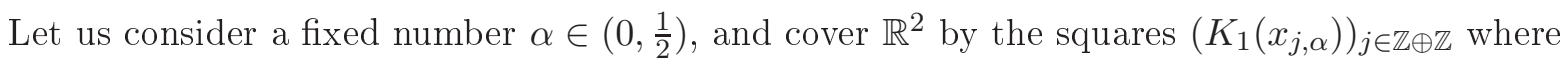

$$
x_{j, \alpha}=(1-\alpha) j, \quad \forall j=\left(j_{1}, j_{2}\right) \in \mathbb{Z} \times \mathbb{Z} .
$$

Let us take a partition of unity in $\mathbb{R}^{2}$ associated with the squares $K_{1}\left(x_{j, \alpha}\right)$,

$$
\sum_{j} u_{j}^{2}=1, \quad \operatorname{supp} u_{j} \subset K_{1}\left(x_{j, \alpha}\right) .
$$

Recall the parameter $\varepsilon=1 / \sqrt{\kappa H}$. Let us consider a further parameter $R=R(\varepsilon)>1$ such that

$$
R(\varepsilon) \rightarrow \infty, \quad \varepsilon R(\varepsilon) \rightarrow 0 \quad \text { as } \varepsilon \rightarrow 0, \quad \text { and } R^{2} \in 2 \pi \mathbb{N} .
$$

Then, defining,

$$
u_{j, R}(x)=u_{j}\left(\frac{x}{\varepsilon R}\right), \quad \forall x \in \Omega,
$$

we get a partition of unity associated with the re-scaled squares $K_{\varepsilon R}\left(x_{j, \alpha}^{\varepsilon}\right)$

$$
x_{j, \alpha}^{\varepsilon}=\varepsilon R x_{j, \alpha}=(1-\alpha) \varepsilon R j, \quad j \in \mathbb{Z} \times \mathbb{Z},
$$

such that,

$$
\operatorname{supp} u_{j, R} \subset K_{\varepsilon R}\left(x_{j, \alpha}^{\varepsilon}\right) \text {. }
$$

In order to simplify notation, we will skip the dependence on $\varepsilon$ and $\alpha$ from the squares and write $K_{\varepsilon R}^{j}$ instead of $K_{\varepsilon R}\left(x_{j, \alpha}^{\varepsilon}\right), j \in \mathbb{Z}^{2}$.

Let us introduce further,

$$
\mathcal{J}=\mathcal{J}_{\varepsilon, D_{a}}=\left\{j: D_{a} \cap \operatorname{supp} u_{j, R} \neq \emptyset\right\}, \quad N_{\varepsilon}=\operatorname{Card} \mathcal{J} .
$$

Here $a=a(\kappa) \rightarrow 0$ as $\kappa \rightarrow \infty$ and $D_{a}$ is the neighborhood of $D$ introduced in (5.1).

We notice that,

$$
\lim _{\varepsilon \rightarrow 0}\left(N_{\varepsilon} \times \varepsilon^{2} R^{2}\right)=(1+v(\alpha))|D|,
$$

where $v(\alpha)$ is positive and verifies (actually $v(\alpha)=\alpha(1-\alpha)$ ),

$$
\lim _{\alpha \rightarrow 0} v(\alpha)=0 .
$$

Implementing the partition of unity $u_{j, R}$, we get a splitting of the interior energy,

$$
\mathcal{E}_{0}\left(\chi_{2, \eta} h \psi, \mathbf{A} ; \Omega\right) \geq \sum_{j \in \mathcal{J}} \mathcal{E}_{0}\left(u_{j, R} \chi_{2, \eta} h \psi, \mathbf{A} ; \Omega\right)-\frac{C}{(\varepsilon R)^{2}}\|\psi\|_{L^{\infty}\left(\left\{x \in \Omega: \operatorname{dist}(x, \partial \Omega) \geq \kappa^{-\rho}\right\}\right)}^{2} .
$$

Setting

$$
\varphi_{j, R}(x)=u_{j, R}(x) \chi_{2, \eta}(x) h(x) \psi(x), \quad \forall x \in \Omega,
$$

and invoking again Theorem 1.4, we infer from (5.28),

$$
\mathcal{E}_{0}\left(\chi_{2, \eta} h \psi, \mathbf{A} ; \Omega\right) \geq \sum_{j \in \mathcal{J}} \mathcal{E}_{0}\left(\varphi_{j, R}, \mathbf{A} ; \Omega\right)-\frac{C}{(\varepsilon R)^{2}} \zeta^{2},
$$

and we point out that the constant $C$ depends on the parameter $\alpha$, but we will not need to make this dependence explicit in the notation as $\alpha$ remains fixed in the limit $\varepsilon \rightarrow 0$. We also remind the reader that $\zeta=\max \left\{\left|1-\frac{\kappa}{H}\right|^{1 / 2}, \kappa^{-1 / 4}\right\}$. 
Let us proceed to estimate $\mathcal{E}_{0}\left(\varphi_{j, R}, \mathbf{A} ; \Omega\right)$. We apply first a gauge transformation that allows us to approximate the vector field $\mathbf{A}$ locally. Setting

$$
\mathbf{B}(x)=\operatorname{curl} \mathbf{A}(x),
$$

then there exists a real-valued function $\phi_{0}$ such that we may write,

$$
\mathbf{A}(x)-\nabla \phi_{0}=\int_{0}^{1} s B(s x)\left(-x_{2}, x_{1}\right) d s, \quad \forall x=\left(x_{1}, x_{2}\right) \in \Omega .
$$

Notice that $\varphi_{j, R}$ is supported in a ball $B\left(x_{j}, C \varepsilon R\right)$ with $C$ sufficiently large. We may write,

$$
\mathbf{A}_{0}(x)-\nabla \phi_{0}=\frac{\mathbf{B}\left(x_{j}\right)}{2}\left(-x_{2}, x_{1}\right)+a(x), \quad \text { in } B\left(x_{j}, C \varepsilon R\right),
$$

where the vector field $a(x)$ satisfies the uniform estimate,

$$
|a(x)| \leq C\|\nabla \mathbf{B}\|_{L^{\infty}(\Omega)}\left|x-x_{j}\right|, \quad \text { in } B\left(x_{j}, C \varepsilon R\right) .
$$

Therefore, we can find a real-valued function $\phi_{j, R, \varepsilon}$ such that,

$$
\mathbf{A}_{0}(x)-\nabla \phi_{j, R, \varepsilon}=\frac{\mathbf{B}\left(x_{j}\right)}{2}\left(x-x_{j}\right)^{\perp}+a(x), \quad \text { in } B\left(x_{j}, C \varepsilon R\right),
$$

where $x^{\perp}=\left(-x_{2}, x_{1}\right)$ for all $x=\left(x_{1}, x_{2}\right) \in \mathbb{R}^{2}$.

By Lemma 2.2, the magnetic field $\mathbf{B}$ is almost equal to 1 , hence we get,

$$
\left|\mathbf{A}(x)-\nabla \phi_{j, R, \varepsilon}(x)-\mathbf{A}_{0}(x)\right| \leq C \kappa^{-1}\left|x-x_{j}\right|, \quad \text { in } B\left(x_{j}, C \varepsilon R\right) .
$$

Here $\mathbf{A}_{0}$ is the vector field introduced in (2.11). Therefore, setting

$$
\mathbf{A}_{j, R}(x)=\mathbf{A}(x)-\nabla \phi_{j, R, \varepsilon}(x),
$$

we infer from (5.30),

$$
\left|\mathbf{A}_{j, R}(x)-\mathbf{A}_{0}(x)\right| \leq C \varepsilon\left|x-x_{j}\right|, \quad \operatorname{in} \operatorname{supp} \varphi_{j, R} .
$$

Furthermore, we notice that,

$$
\mathcal{E}_{0}\left(\varphi_{j, R}, \mathbf{A} ; \Omega\right)=\mathcal{E}_{0}\left(e^{i \phi_{j, R, \varepsilon}} \varphi_{j, R}, \mathbf{A}_{j, R} ; \Omega\right) .
$$

Using a Cauchy-Schwarz inequality, we get for any $\beta \in(0,1)$,

$$
\begin{aligned}
\mathcal{E}_{0}\left(e^{i \phi_{j, R, \varepsilon}} \varphi_{j, R}, \mathbf{A}_{j, R} ; \Omega\right) \geq(1-\beta) \mathcal{E}_{0}\left(e^{i \phi_{j, R, \varepsilon}} \varphi_{j, R}, \mathbf{A}_{0} ; \Omega\right) & \\
& -C \beta^{-1} \varepsilon^{-4} \int_{\Omega}\left|\mathbf{A}_{j, R}(x)-\mathbf{A}_{0}(x)\right|^{2}\left|\varphi_{j, R}\right|^{2} d x .
\end{aligned}
$$

We implement (5.31) in the above estimate and we use the bound $\left|\varphi_{j, \eta}(x)\right| \leq \zeta$. That way we get,

$$
\mathcal{E}_{0}\left(e^{i \phi_{j, R, \varepsilon}} \varphi_{j, R}, \mathbf{A}_{j, R} ; \Omega\right) \geq(1-\beta) \mathcal{E}_{0}\left(e^{i \phi_{j, R, \varepsilon}} \varphi_{j, R}, \mathbf{A}_{0} ; \Omega\right)-C \beta^{-1} \varepsilon^{2} R^{4} \zeta^{2} .
$$

We proceed to obtain a lower bound for $\mathcal{E}_{0}\left(e^{i \phi_{j, R, \varepsilon}} \varphi_{j, R}, \mathbf{A}_{0} ; \Omega\right)$. Modulo a translation, we may assume that $\operatorname{supp} \varphi_{j, R}$ is contained in the unit square $K_{\varepsilon R}$ of the lattice $\varepsilon R(\mathbb{Z} \oplus i \mathbb{Z})$. We therefore define the re-scaled function,

$$
f_{j}(\widetilde{x})=\left(e^{i \phi_{j, R, \varepsilon}} \varphi_{j, R}\right)(\varepsilon \widetilde{x}), \quad \forall \widetilde{x} \in K_{R}
$$

That way the energy becomes (after omitting the tildes from the notation),

$$
\mathcal{E}_{0}\left(e^{i \phi_{j, R, \varepsilon}} \varphi_{j, R}, \mathbf{A}_{0} ; \Omega\right)=\int_{K_{R}}\left(\left|\left(\nabla-i \mathbf{A}_{0}\right) f_{j}\right|^{2}-\frac{\kappa}{H}\left|f_{j}\right|^{2}+\frac{\kappa}{2 H}\left|f_{j}\right|^{4}\right) d x .
$$

Invoking (5.32) and (5.33), we deduce that,

$$
\begin{aligned}
\mathcal{E}_{0}\left(\varphi_{j, R}, \mathbf{A} ; \Omega\right)=(1-\beta) \int_{K_{R}}\left(\left|\left(\nabla-i \mathbf{A}_{0}\right) f_{j}\right|^{2}-\frac{\kappa}{H}\left|f_{j}\right|^{2}+\frac{\kappa}{2 H}\left|f_{j}\right|^{4}\right) d x & \\
& -C \beta^{-1} \varepsilon^{2} R^{4} \zeta^{2} .
\end{aligned}
$$


Up to now, we are able to prove the following lemma, whose proof is actually a simple application of the Cauchy-Schwarz inequality.

Lemma 5.3. Assume that $H=\kappa-\mu(\kappa) \sqrt{\kappa}$ with $\lim \sup \mu(\kappa)=\mu_{0}$ and $\mu_{0} \in[-\infty, 0]$. Then, as $\kappa \rightarrow \infty$,

$$
\mathcal{E}_{0}\left(\chi_{2, \eta} h \psi, \mathbf{A} ; \Omega\right) \geq-C\left(\beta^{-1} R^{2}+\frac{1}{(\varepsilon R)^{2}}\right) \zeta^{2}+o(\kappa) .
$$

Proof. Notice that, for any $j, f_{j}$ can be considered as a function in the domain of the periodic operator $P_{R}$, see (2.15). Using Proposition 2.7 and the variational min-max principle, we write,

$$
\begin{aligned}
\int_{K_{R}}\left(\left|\left(\nabla-i \mathbf{A}_{0}\right) f_{j}\right|^{2}-\frac{\kappa}{H}\left|f_{j}\right|^{2}+\frac{\kappa}{2 H}\left|f_{j}\right|^{4}\right) & d x \\
& \geq\left(1-\frac{\kappa}{H}\right) \int_{K_{R}}\left|f_{j}\right|^{2} d x+\frac{\kappa}{2 H} \int_{K_{R}}\left|f_{j}\right|^{4} d x .
\end{aligned}
$$

If $\mu(\kappa) \leq 0$, i.e. $H \geq \kappa$, we have nothing to prove since the right hand side of (5.36) is positive, and we only need to collect the estimates (5.29) and (5.32)-(5.35).

Now we assume that $\mu(\kappa) \rightarrow 0$ as $\kappa \rightarrow \infty$. Using the following Cauchy-Schwarz inequality

$$
|\mu(\kappa)| \sqrt{\kappa} \int_{K_{R}}\left|f_{j}\right|^{2} d x \leq \frac{1}{4} \kappa \int_{K_{R}}\left|f_{j}\right|^{4}+4 R^{2} \mu^{2}(\kappa),
$$

we obtain from (5.36),

$$
\begin{aligned}
\int_{K_{R}}\left(\left|\left(\nabla-i \mathbf{A}_{0}\right) f_{j}\right|^{2}-\frac{\kappa}{H}\left|f_{j}\right|^{2}+\frac{\kappa}{2 H}\left|f_{j}\right|^{4}\right) d x & \geq \frac{\kappa}{4 H} \int_{K_{R}}\left|f_{j}\right|^{4} d x-\frac{4 R^{2} \mu^{2}(\kappa)}{H} \\
& \geq-\frac{5 R^{2} \mu^{2}(\kappa)}{H} .
\end{aligned}
$$

Summing over $j$ (recall that the number of indices $j$ is proportional to $\varepsilon^{-2} R^{-2} \sim \kappa^{2} R^{-2}$ ), we get

$$
\sum_{j}\left(\left|\left(\nabla-i \mathbf{A}_{0}\right) f_{j}\right|^{2}-\frac{\kappa}{H}\left|f_{j}\right|^{2}+\frac{\kappa}{2 H}\left|f_{j}\right|^{4}\right) d x \geq-C \mu^{2}(\kappa) \kappa .
$$

To finish the proof of the lemma, it suffices to collect the estimates (5.29) and (5.32)

We assume from now on that $H=\kappa-\mu(\kappa) \sqrt{\kappa}$ with

$$
\mu(\kappa)>0 \text {. }
$$

Let us introduce,

$$
\mathcal{J}_{+}=\left\{j \in \mathcal{J}: \int_{K_{R}^{j}}\left(\left|\left(\nabla-i \mathbf{A}_{0}\right) f_{j}\right|^{2}-\frac{\kappa}{H}\left|f_{j}\right|^{2}\right) d x \geq 0\right\},
$$

and set

$$
n_{\varepsilon}=\operatorname{Card} \mathcal{J}_{+} .
$$

We shall obtain a lower bound of $\mathcal{E}_{0}\left(\chi_{2, \eta} h \psi, \mathbf{A} ; \Omega\right)$ in terms of the 'local energies' associated with the indices $j$ that are not in $\mathcal{J}_{+}$.

Let us pick an arbitrary $j \notin \mathcal{J}_{+}$. Then,

$$
\int_{K_{R}}\left(\left|\left(\nabla-i \mathbf{A}_{0}\right) f_{j}\right|^{2}-\frac{\kappa}{H}\left|f_{j}\right|^{2}\right) d x<0 .
$$

Notice that the function $f_{j}$ belongs to the domain of the periodic operator $P_{R}$, whose first eigenvalue equals to 1 . Let us recall that we introduced the following parameter

$$
\gamma=\frac{\kappa}{H}-1
$$


With this in hand we may write,

$$
Q_{R}\left(f_{j}\right)-(1+\gamma) \int_{K_{R}}\left|f_{j}\right|^{2} d x<0 .
$$

Invoking Lemma 2.8, we get,

$$
\left\|f_{j}-\Pi_{1} f_{j}\right\|_{L^{4}\left(K_{R}\right)} \leq C \sqrt{\gamma}\left\|f_{j}\right\|_{L^{2}\left(K_{R}\right)} .
$$

Here, recall the space $L_{R}$ introduced in (2.22) and $\Pi_{1}$ the orthogonal projector on $L_{R}$.

It results from the triangle inequality and (5.38) that

$$
\left\|\Pi_{1} f_{j}\right\|_{L^{4}\left(K_{R}\right)} \leq\left\|f_{j}\right\|_{L^{4}\left(K_{R}\right)}+C \sqrt{\gamma}\left\|f_{j}\right\|_{L^{2}\left(K_{R}\right)} .
$$

Applying Cauchy-Schwarz inequality twice, we get for any $\sigma \in(0,1)$ the following estimate,

$$
\left\|\Pi_{1} f_{j}\right\|_{L^{4}\left(K_{R}\right)}^{4} \leq(1+\sigma)\left\|f_{j}\right\|_{L^{4}\left(K_{R}\right)}^{4}+C \sigma^{-3} \gamma^{2}\left\|f_{j}\right\|_{L^{2}\left(K_{R}\right)}^{4} .
$$

Using the definition of $f_{j}$ and Theorem 1.4, we get that $\left|f_{j}\right| \leq \zeta$. Hence, we infer from (5.39),

$$
\left\|\Pi_{1} f_{j}\right\|_{L^{4}\left(K_{R}\right)}^{4} \leq(1+\sigma)\left\|f_{j}\right\|_{L^{4}\left(K_{R}\right)}^{4}+C \sigma^{-3} \gamma^{2} R^{4} \zeta^{4} .
$$

Using the mini-max principle and (5.40), we get,

$$
\begin{aligned}
\int_{K_{R}} & \left(\left|\left(\nabla-i \mathbf{A}_{0}\right) f_{j}\right|^{2}-\frac{\kappa}{H}\left|f_{j}\right|^{2}+\frac{\kappa}{2 H}\left|f_{j}\right|^{4}\right) d x \\
& \geq \int_{K_{R}}\left[\left(1-\frac{\kappa}{H}\right)\left|\Pi_{1} f_{j}\right|^{2}+\frac{\kappa}{2 H}(1-\sigma)\left|\Pi_{1} f_{j}\right|^{4}\right] d x-C \sigma^{-3} \gamma^{2} R^{4} \zeta^{2} .
\end{aligned}
$$

We choose $\sigma$ as function of $\varepsilon$ and we impose on it the following condition,

$$
\sigma \rightarrow 0 \quad \text { as } \varepsilon \rightarrow 0
$$

Therefore, defining

$$
c=\sqrt{\frac{\frac{\kappa}{H}(1-\sigma)}{\frac{\kappa}{H}-1}}, \quad g(x)=c \Pi_{1} f_{j},
$$

we get,

$$
\begin{aligned}
\int_{K_{R}}\left(\left|\left(\nabla-i \mathbf{A}_{0}\right) f_{j}\right|^{2}-\frac{\kappa}{H}\left|f_{j}\right|^{2}+\frac{\kappa}{2 H}\left|f_{j}\right|^{4}\right) d x & =\frac{\left|K_{R}\right|}{c^{2}}\left(\frac{\kappa}{H}-1\right) F_{R}(g) \\
& \geq\left|K_{R}\right| \frac{|\mu(\kappa)|^{2}}{\kappa}(1+o(1)) c(R) \quad \text { as } \kappa \rightarrow \infty .
\end{aligned}
$$

Here recall the energy $F_{R}$ and the constant $c(R)$ introduced in (2.21) and (2.23) respectively.

Consequently, collecting (5.29), (5.32), (5.33), (5.35), (5.42), we get,

$$
\begin{aligned}
\mathcal{E}_{0}\left(\chi_{2, \eta} h \psi, \mathbf{A} ; \Omega\right) \geq & (1-\beta)\left[\left(N_{\varepsilon}-n_{\varepsilon}\right) \frac{\left|K_{R}\right|}{\kappa}|\mu(\kappa)|^{2} c(R)(1+o(1))+\sum_{j \in \mathcal{J}_{+}} \frac{\kappa}{2 H} \int\left|f_{j}\right|^{4} d x\right] \\
& -C\left[\left(\beta^{-1} R^{2}+\frac{1}{(\varepsilon R)^{2}}\right) \zeta^{2}+\sigma^{-3} \varepsilon^{-2} \gamma^{2} R^{2} \zeta^{4}\right] .
\end{aligned}
$$

Notice that, as a result of (5.26), we have,

$$
\frac{N_{\varepsilon}\left|K_{R}\right|}{\kappa}=\kappa c_{\alpha}|D|+o(\kappa) \text { as } \kappa \rightarrow \infty, \quad \text { and } \quad c(R)=-E_{2}+o(1) \text { as } R \rightarrow \infty,
$$


where $c_{\alpha}=1+\alpha(1-\alpha)$ and the constant $\alpha \in(0,1)$ is introduced in connection with the partition of unity $u_{j, R}$. Since $E_{2}>0$, we deduce the following lower bound from (5.43),

$$
\begin{aligned}
\mathcal{E}_{0}\left(\chi_{2, \eta} h \psi, \mathbf{A} ; \Omega\right) \geq & (1-\beta)\left(-c_{\alpha}|D| E_{2}[\mu(\kappa)]_{+}^{2} \kappa+o\left(|\mu(\kappa)|^{2} \kappa\right)\right) \\
& -C\left[\left(\beta^{-1} R^{2}+\frac{1}{(\varepsilon R)^{2}}\right) \zeta^{2}+\sigma^{-3} \varepsilon^{-2} \gamma^{2} R^{2} \zeta^{4}\right] .
\end{aligned}
$$

5.4. Proof of Theorem 5.1. We recall that we only treat the case when the domain $D$ satisfies the condition in (5.4). We also recall the condition $\liminf _{\kappa \rightarrow \infty} \mu(\kappa)>-\infty$.

Assume that the conclusion of Theorem 5.1 were false. Then there exist sequences $\left\{\kappa_{n}\right\},\left\{H_{n}\right\}$, $\mu_{0} \in \mathbb{R} \cup\{+\infty\}, c>0$ and a sequence of minimizers $\left\{\left(\psi_{n}, \mathbf{A}_{n}\right)\right\}$ of the energy $\mathcal{E}$ such that,

$$
\kappa_{n} \rightarrow \infty \quad \frac{\kappa_{n}}{H_{n}} \rightarrow 1 \quad \mu\left(\kappa_{n}\right) \rightarrow \mu_{0} \quad \text { as } n \rightarrow \infty,
$$

and

$$
\mathcal{E}\left(h \psi_{n}, \mathbf{A}_{n} ; \Omega\right) \leq-E_{1}|\Omega| \kappa_{n}-E_{2}|D|\left[\mu\left(\kappa_{n}\right)\right]_{+}^{2} \kappa_{n}-c \max \left(1,\left[\mu\left(\kappa_{n}\right)\right]_{+}^{2}\right) \kappa_{n} .
$$

We treat seperately the two cases $\mu_{0} \in(-\infty, 0]$ and $\mu_{0} \in(0, \infty]$. Assume that $\mu_{0} \in(-\infty, 0]$. Then $\zeta=\max \left\{\left|1-\frac{\kappa_{n}}{H_{n}}\right|^{1 / 2}, \kappa_{n}^{-1 / 4}\right\}=\kappa_{n}^{-1 / 4}$. Combining (5.11), (5.21) and Lemma 5.3, we get,

$$
\begin{aligned}
\mathcal{E}_{0}\left(h \psi_{n}, \mathbf{A}_{n} ; \Omega\right) \geq & -E_{1}|\partial \Omega| \kappa_{n}-C\left[\kappa_{n}^{2}\left(\eta^{2}+\eta \frac{\left|\mu\left(\kappa_{n}\right)\right|}{\sqrt{\kappa_{n}}}+\delta^{-1} \kappa_{n}^{2} \eta^{5}\right)+(\delta+\eta) \kappa_{n}+\frac{1}{\eta} \kappa_{n}^{-1 / 2}\right] \\
& -C\left(\beta^{-1} R^{2}+\frac{1}{\left(\varepsilon_{n} R\right)^{2}}\right) \varepsilon_{n}^{1 / 2}+o\left(\kappa_{n}\right) .
\end{aligned}
$$

Here $\varepsilon_{n}=\frac{1}{\sqrt{\kappa_{n} H_{n}}}$. We choose

$$
\begin{gathered}
\eta=\kappa_{n}^{-\rho}, \quad \delta=\sqrt{\kappa_{n}^{3} \eta^{5}}, \quad \frac{3}{5}<\rho<1, \\
\beta=\kappa_{n}^{-1 / 4},
\end{gathered}
$$

and $R=2 \pi\left[\kappa_{n}^{3 / 4}\right]$, with $[x]$ denotes the integer part of $x$. In this way we get,

$$
\mathcal{E}_{0}\left(h \psi_{n}, \mathbf{A}_{n} ; \Omega\right) \geq-E_{1}|\partial \Omega| \kappa_{n}+o\left(\kappa_{n}\right)
$$

thereby contradicting (5.45).

We now treat the case $\mu_{0} \in(0, \infty]$. In this case $\zeta \approx \sqrt{\mu\left(\kappa_{n}\right)} \kappa_{n}^{-1 / 4}$ and $\gamma=\frac{\kappa_{n}}{H_{n}}-1 \approx \zeta^{2}$.

We make the following choice of the parameters:

$$
\begin{gathered}
\eta=\mu^{2 / 5}\left(\kappa_{n}\right) \kappa_{n}^{-\rho}, \quad \delta=\frac{\sqrt{\kappa_{n}^{3} \eta^{5}}}{\mu\left(\kappa_{n}\right)}, \quad \frac{3}{5}<\rho<1, \\
\beta=\left(\mu_{n}^{2} \varepsilon_{n}\right)^{3 / 8}, \quad \sigma=\left(\mu_{n}^{2} \varepsilon_{n}\right)^{1 / 16},
\end{gathered}
$$

and $R=2 \pi\left[\left(\mu_{n}^{2} \varepsilon_{n}\right)^{-3 / 8}\right]$. Here $\mu_{n}=\mu\left(\kappa_{n}\right)$. With this choice of parameters, we have,

$$
\frac{1}{\left(\varepsilon_{n} R\right)^{2}} \zeta^{2}+\beta^{-1} R^{2} \zeta^{2}+\sigma^{-3} \varepsilon_{n}^{-2} \gamma^{2} R^{2} \zeta^{4}=o\left(\mu^{2} \varepsilon_{n}^{-1}\right) \quad \text { as } \varepsilon_{n} \rightarrow 0 .
$$

Therefore, we get by combining (5.11), (5.21) and (5.44),

$$
\mathcal{E}_{0}\left(h \psi_{n}, \mathbf{A}_{n} ; \Omega\right) \geq-E_{1}|\partial \Omega| \kappa_{n}-c_{\alpha} E_{2}|D|\left[\mu\left(\kappa_{n}\right)\right]^{2} \kappa_{n}+o\left(\mu_{n}^{2} \kappa_{n}\right) .
$$

Actually, we have proved the following lower bound,

$$
\liminf _{n \rightarrow \infty} \frac{\mathcal{E}_{0}\left(h \psi_{n}, \mathbf{A}_{n} ; \Omega\right)}{\mu^{2}\left(\kappa_{n}\right) \kappa_{n}} \geq \begin{cases}-E_{2}|D| c_{\alpha} & \text { if } \mu_{0}=+\infty \\ -E_{1}|\partial \Omega| \mu_{0}^{-2}-E_{2}|D| c_{\alpha} & \text { if } \mu_{0} \in(0, \infty)\end{cases}
$$


Since the term on the left side in (5.46) is independent from $\alpha$, we get by taking $\alpha \rightarrow 0_{+}$on both sides (recall that $c_{\alpha}=1+\alpha(1-\alpha)$ ),

$$
\liminf _{n \rightarrow \infty} \frac{\mathcal{E}_{0}\left(h \psi_{n}, \mathbf{A}_{n} ; \Omega\right)}{\mu^{2}\left(\kappa_{n}\right) \kappa_{n}} \geq \begin{cases}-E_{2}|D| & \text { if } \mu_{0}=\infty \\ -E_{1}|\partial \Omega| \mu_{0}^{-2}-E_{2}|D| & \text { if } \mu_{0} \in(0, \infty),\end{cases}
$$

which contradicts the upper bound in (5.45). Therefore, the conclusion of Theorem 5.2 holds true.

Recalling that $\mathcal{E}(\psi, \mathbf{A} ; \Omega)=\mathcal{E}_{0}(\psi, \mathbf{A} ; \Omega)+(\kappa H)^{2} \int_{\Omega}|\operatorname{curl} \mathbf{A}-1|^{2} d x$, we get as an immediate corollary of Theorem 4.1 and the proof of Theorem 5.1 .

Corollary 5.4. Assume that $H=\kappa-\mu(\kappa) \sqrt{\kappa}$ with $\lim _{\kappa \rightarrow \infty} \frac{\mu(\kappa)}{\sqrt{\kappa}}=0$. Then, for any minimizer $(\psi, \mathbf{A})$ of (1.1), the following asymptotic estimate holds:

$$
\kappa^{2} H^{2} \int_{\Omega}|\operatorname{curl} \mathbf{A}-1|^{2} d x=o\left(\max \left([\mu(\kappa)]_{+}^{2}, 1\right) \kappa\right) \quad \text { as } \kappa \rightarrow \infty .
$$

\section{Proof of the energy estimates}

We proceed in this section to complete the proofs of Theorem 1.2 and Corollary 1.3 We start by a localization estimate.

Lemma 6.1. Assume $H=\kappa-\mu(\kappa) \sqrt{\kappa}$ such that

$$
\lim _{\kappa \rightarrow \infty} \frac{\mu(\kappa)}{\sqrt{\kappa}}=0 .
$$

Then, for any minimizer $(\psi, \mathbf{A})$ of (1.1) and any open, smooth domain $D \subset \Omega$, we have as $\kappa \rightarrow \infty$ :

$$
\begin{aligned}
\mathcal{E}(\psi, \mathbf{A} ; D)-\mathcal{E}(f \psi, \mathbf{A} ; D)-\int_{D}|\nabla f|^{2}|\psi|^{2} d x & +\frac{\kappa^{2}}{2} \int_{D}\left(1-f^{2}\right)^{2}|\psi|^{4} d x \\
& =-\operatorname{Re} \int_{\partial \Omega}|\psi|^{2} \bar{f} \nu \cdot \nabla f d \sigma+o(\kappa) .
\end{aligned}
$$

Here $\nu$ is the unit inward normal vector of $\partial \Omega$ and $f$ is any function such that,

Proof of Lemma 6.1.

$$
\nabla f \in L^{\infty}\left(\mathbb{R}^{2}\right), \quad \operatorname{supp} f \subset \bar{D} .
$$

Integrating by parts, we get the following localization formula,

$$
\begin{aligned}
\int_{\Omega}|(\nabla-i \kappa H) f \psi|^{2} d x= & \operatorname{Re} \int_{\Omega}-(\nabla-i \kappa H \mathbf{A})^{2} \psi \overline{f^{2} \psi} d x \\
& -\operatorname{Re} \int_{\partial \Omega}|\psi|^{2} \bar{f} \nu \cdot \nabla f d \sigma+\int_{\Omega}|\nabla f|^{2}|\psi|^{2} d x .
\end{aligned}
$$

Using the equation for $\psi$ in (2.1) and the assumption $\operatorname{supp} f \subset \bar{D}$, we get further,

$$
\begin{array}{r}
\mathcal{E}(f \psi, \mathbf{A} ; D)=\kappa^{2} \int_{\Omega}\left(\frac{1}{2} f^{2}-1\right) f^{2}|\psi|^{4} d x+\int_{\Omega}|\nabla f|^{2}|\psi|^{2} d x-\operatorname{Re} \int_{\partial \Omega}|\psi|^{2} \bar{f} \nu \cdot \nabla f d \sigma \\
+(\kappa H)^{2} \int_{D}|\operatorname{curl} \mathbf{A}-1|^{2} d x .
\end{array}
$$

Similarly, we get,

$$
\begin{aligned}
\mathcal{E}(\psi, \mathbf{A} ; D)=-\frac{\kappa^{2}}{2} \int_{D}|\psi|^{4} d x+(\kappa H)^{2} \int_{D}|\operatorname{curl} \mathbf{A}-1|^{2} d x & \\
& +\int_{\bar{D} \cap \partial \Omega} \bar{\psi} \nu \cdot(\nabla-i \kappa H \mathbf{A}) \psi d \sigma+\int_{\Omega \cap \partial D} \bar{\psi} \nu \cdot(\nabla-i \kappa H \mathbf{A}) \psi d \sigma .
\end{aligned}
$$


Combining (6.3) and (6.4), we need only establish that

as $\kappa \rightarrow \infty$.

$$
\int_{\bar{D} \cap \partial \Omega} \bar{\psi} \nu \cdot(\nabla-i \kappa H \mathbf{A}) \psi d \sigma+\int_{\Omega \cap \partial D} \bar{\psi} \nu \cdot(\nabla-i \kappa H \mathbf{A}) \psi d \sigma=o(\kappa),
$$

Thanks to the boundary condition in (2.1), the integral over $\bar{D} \cap \partial \Omega$ vanishes. So we only consider the integral over $\Omega \cap \partial D$. To that end we write,

$$
\begin{aligned}
\int_{\Omega \cap \partial D} \bar{\psi} \nu \cdot(\nabla-i \kappa H \mathbf{A}) \psi d \sigma= & \left.\int_{\{x \in \Omega \cap \partial D}: \operatorname{dist}(x, \partial \Omega) \leq g_{1}(\kappa)\right\} \\
& \bar{\psi} \nu \cdot(\nabla-i \kappa H \mathbf{A}) \psi d \sigma \\
& \int_{\left\{x \in \Omega \cap \partial D: \operatorname{dist}(x, \partial \Omega) \geq g_{1}(\kappa)\right\}} \bar{\psi} \nu \cdot(\nabla-i \kappa H \mathbf{A}) \psi d \sigma .
\end{aligned}
$$

Here $g_{1}(\kappa)$ is any positive function such that $g_{1}(\kappa) \rightarrow 0$ and $\kappa g_{1}(\kappa) \rightarrow \infty$ as $\kappa \rightarrow \infty$. Invoking the results of Lemmas 2.2 and 2.3 , we deduce that

$$
\int_{\Omega \cap \partial D} \bar{\psi} \nu \cdot(\nabla-i \kappa H \mathbf{A}) \psi d \sigma=o(\kappa) \quad \text { as } \kappa \rightarrow \infty .
$$

\section{Proof of Theorem 1.2.}

Thanks to Theorem 1.1, we may assume the condition $\liminf _{\kappa \rightarrow \infty} \mu(\kappa)>-\infty$.

Let us consider a partition of unity on $\mathbb{R}$,

$$
h_{1}^{2}+h_{2}^{2}=1 \quad \text { in } \mathbb{R}, \quad \operatorname{supp} h_{1} \subset(-1, \infty), \quad \operatorname{supp} h_{2} \subset\left(-\infty,-\frac{1}{2}\right) .
$$

Let $m=m(\kappa) \in(0,1)$ be a parameter that will be specified later. Define the 'signed' distance to the boundary between $D$ and $\Omega \backslash \bar{D}$,

$$
t_{D}(x)=\left\{\begin{array}{ll}
-\operatorname{dist}(x, \Gamma) & \text { if } x \in D, \\
\operatorname{dist}(x, \Gamma) & \text { if } x \notin D .
\end{array}, \quad \text { with } \Gamma:=\partial D \backslash \partial \Omega .\right.
$$

For any $x \in \bar{\Omega}$, we define,

$$
\varphi_{1, m}(x)=h_{1}\left(\frac{t_{D}(x)}{m}\right) \psi(x), \quad \varphi_{2, m}(x)=h_{2}\left(\frac{t_{D}(x)}{m}\right) \psi(x) .
$$

Then, it results from the IMS decomposition formula,

$$
\mathcal{E}(\psi, \mathbf{A} ; \Omega) \geq \mathcal{E}\left(\varphi_{1, m}, \mathbf{A} ; \Omega\right)+\mathcal{E}\left(\varphi_{2, m}, \mathbf{A} ; \Omega\right)-\frac{C}{m^{2}} \int_{\Omega}|\psi|^{2} d x .
$$

We shall assume the following condition on $m=m(\kappa)$,

$$
m \ll 1 \quad \text { and } m^{-1}+m^{-2} \zeta^{2} \ll \max \left(1,[\mu(\kappa)]_{+}^{2}\right) \kappa \quad \text { as } \kappa \rightarrow \infty .
$$

Here $\zeta(\kappa)=\max \left\{\left|1-\frac{\kappa}{H}\right|^{1 / 2}, \kappa^{-1 / 4}\right\}$ as previously. The choice $m=\frac{1}{\sqrt{\kappa}}$ fulfills the condition in (6.7).

Invoking Corollary 3.1 and the upper bound of Theorem 4.1, we get under the condition (6.7),

$$
\mathcal{E}\left(\varphi_{1, m}, \mathbf{A} ; \Omega\right)+\mathcal{E}\left(\varphi_{2, m}, \mathbf{A} ; \Omega\right) \leq-\mathcal{A}(\mu(\kappa) ; \Omega)+o\left(\max \left(1,[\mu(\kappa)]_{+}^{2}\right) \kappa\right),
$$

where, for a subdomain $V \subset \Omega$, we define,

$$
\mathcal{A}(\mu(\kappa) ; V)=\left(E_{1}|\bar{V} \cap \partial \Omega|+[\mu(\kappa)]_{+}^{2} E_{2}|V|\right) \kappa .
$$

Notice that $\varphi_{1, m}$ has support in $\bar{U}_{m}$, where

$$
\left.U_{m}=\{x \in \Omega: \operatorname{dist}(x, U))<m\right\}, \quad U=\Omega \backslash \bar{D} .
$$

Applying Theorem 5.1, we get the following lower bound,

$$
\mathcal{E}\left(\varphi_{1, m}, \mathbf{A} ; \Omega\right) \geq-\mathcal{A}(\mu(\kappa) ; \Omega \backslash \bar{D})+o\left(\max \left(1,[\mu(\kappa)]_{+}^{2}\right) \kappa\right) \quad \text { as } \kappa \rightarrow \infty .
$$


Substituting (6.10) in (6.8), we also get the following upper bound

$$
\mathcal{E}\left(\varphi_{2, m}, \mathbf{A} ; \Omega\right) \leq-\mathcal{A}(\mu(\kappa) ; D)+o\left(\max \left(1,[\mu(\kappa)]_{+}^{2}\right) \kappa\right) .
$$

In order to finish the proof of Theorem 1.2, it is sufficient to show for an arbitrary smooth domain $D \subset \Omega$,

$$
\mathcal{E}(\psi, \mathbf{A} ; D) \leq-\mathcal{A}(\mu(\kappa), D)+o\left(\max \left(1,[\mu(\kappa)]_{+}^{2}\right) \kappa\right) \quad \text { as } \kappa \rightarrow \infty,
$$

and

$$
\mathcal{E}(\psi, \mathbf{A} ; \Omega \backslash D) \geq-\mathcal{A}(\mu(\kappa), \Omega \backslash D)+o\left(\max \left(1,[\mu(\kappa)]_{+}^{2}\right) \kappa\right) \quad \text { as } \kappa \rightarrow \infty .
$$

Let us prove (6.12). Notice that $\varphi_{2, m}$ has support in $\bar{D}$. Invoking Lemma 6.1 together with Corollary [3.1, we get (thanks in particular to the condition (6.7) on $\mathrm{m}$ ),

$$
\mathcal{E}(\psi, \mathbf{A} ; D) \leq \mathcal{E}\left(\varphi_{2, m}, \mathbf{A} ; D\right)+o\left(\max \left(1,[\mu(\kappa)]_{+}^{2}\right) \kappa\right) .
$$

Using (6.11), we see that (6.12) actually holds.

Let us prove now (6.13). We have the natural decomposition of the energy,

$$
\mathcal{E}(\psi, \mathbf{A} ; \Omega)=\mathcal{E}(\psi, \mathbf{A} ; D)+\mathcal{E}(\psi, \mathbf{A} ; \Omega \backslash D) .
$$

Using the lower bound in Theorem 5.2, we deduce that,

$$
\mathcal{E}(\psi, \mathbf{A} ; D)+\mathcal{E}(\psi, \mathbf{A} ; \Omega \backslash D) \geq-\mathcal{A}(\mu(\kappa), \Omega)+o\left(\max \left(1,[\mu(\kappa)]_{+}^{2}\right) \kappa\right) .
$$

Inserting the established upper bound (6.12) in the left side above, we get the lower bound in (6.13).

Proof of Corollary 1.3.

Consider any open domain $D \subset \Omega$. Let us multiply the G-L equation (2.1) for $\psi$ by $\bar{\psi}$ and integrate over $\Omega$. Integrating by parts and using Corollary 5.4, we obtain,

$$
\begin{aligned}
\mathcal{E}(\psi, \mathbf{A} ; D)=-\frac{\kappa^{2}}{2} \int_{D}|\psi|^{4} d x+\int_{\bar{\Omega} \cap \partial D} \bar{\psi} \nu_{D} \cdot(\nabla-i \kappa H \mathbf{A}) \psi d \sigma & \\
& +o\left(\max \left(1,[\mu(\kappa)]_{+}^{2}\right) \kappa\right), \quad \text { as } \kappa \rightarrow \infty .
\end{aligned}
$$

Using Lemmas 2.2 and 2.3, we get (see the proof of (6.5)),

$$
\int_{\bar{\Omega} \cap \partial D} \bar{\psi} \nu_{D} \cdot(\nabla-i \kappa H \mathbf{A}) \psi d \sigma=o(\kappa) \quad \text { as } \kappa \rightarrow \infty .
$$

In particular we have,

$$
\mathcal{E}(\psi, \mathbf{A} ; D)=-\frac{\kappa^{2}}{2} \int_{D}|\psi|^{4} d x+o\left(\max \left(1,[\mu(\kappa)]_{+}^{2}\right) \kappa\right), \quad \text { as } \kappa \rightarrow \infty .
$$

Implementing the asymptotic expansion of Theorem 1.2, we obtain,

$$
\frac{\kappa^{2}}{2} \int_{D}|\psi|^{4} d x=\mathcal{A}(\mu(\kappa), D)+o\left(\max \left(1,[\mu(\kappa)]_{+}^{2}\right) \kappa\right), \quad \text { as } \kappa \rightarrow \infty .
$$

Coming back to the definition of $\mathcal{A}(\mu(\kappa), D)$ in (6.9), we get the result of Corollary 1.3.

\section{ACKNOWLEDGEMENTS}

The authors were supported by the European Research Council under the European Community's Seventh Framework Programme (FP7/2007-2013)/ERC grant agreement $n^{\circ} 202859$. SF is also supported by the Danish Research Council and the Lundbeck Foundation. 


\section{REFERENCES}

[1] A. Aftalion. S. Serfaty. Lowest Landau level approach in superconductivity for the Abrikosov lattice close to $H_{c_{2}}$. Selecta Math. (N.S.) 13 (2007), no. 2, 183-202.

[2] Y. Almog. Abrikosov lattices in finite domains. Commun. Math. Phys. 262 (2006), 677-702.

[3] Y. Almog. Non-linear surface superconductivity in the large $\kappa$ limit. Rev. Math. Phys. 16 (2004), 961-976.

[4] Y. Almog, B. Helffer. The distribution of surface superconductivity along the boundary: on a conjecture of X. B. Pan. SIAM J. Math. Anal. 38 (2007), no. 6, 1715-1732.

[5] S. Fournais, B. Helffer. Bulk superconductivity in type II superconductors near the second critical field. To appear in J. Europ. Math. Soc. (JEMS) (2008).

[6] S. Fournais, B. Helffer. Spectral Methods in surface superconductivity. Monograph in press.

[7] S. Fournais, B. Helffer. Strong diamagnetism for general domains and application. Ann. Inst. Fourier 57 (7) 2389-2400 (2007).

[8] S. Fournais, B. Helffer. Optimal uniform elliptic estimates for the Ginzburg-Landau system. Adventures in Mathematical Physics. Contemp. Math. 447 (2007), 83-102.

[9] B. Helffer, X.-B. Pan. Upper critical field and location of surface nucleation of superconductivity. Ann. Inst. H. Poincaré Anal. Non. Linéaire 20 (1) 145-181 (2003).

[10] K. Lu, X.B. Pan. Estimates of the upper critical field for the Ginzburg-Landau equations of superconductivity. Physica D 127 (1999), 73-104.

[11] S. Fournais, B. Helffer. Energy asymptotics for type II superconductors. Calc. Var. Partial Differential Equations 24 (2005), no. 3, 341-376.

[12] X.B. Pan. Surface superconductivity in applied magnetic fields above $H_{C_{2}}$. Commun. Math. Phys. 228 (2002), 228-370.

[13] E. Sandier, S. Serfaty. Vortices for the Magnetic Ginzburg-Landau Model. Progress in Nonlinear Differential Equations and their Applications, 70. Birkhäuser Boston, Inc., Boston, MA, 2007.

[14] E. Sandier, S. Serfaty. The decrease of bulk superconductivity close to the second critical field in the Ginzburg-Landau model. SIAM. J. Math. Anal. 34 No. 4 (2003), 939-956.

(S. Fournais and A. Kachmar) Department of Mathematical Sciences, University of Aarhus, Ny Munkegade, Building 1530, DK-8000 Århus C, Denmark

E-mail address, S. Fournais: fournais@imf .au.dk

E-mail address, A. Kachmar: ayman.kachmar@math.u-psud.fr 